\title{
Quantitative Model for the Prediction of Hydrodynamic Size of Nonionic Reverse Micelles
}

Melissa A. Michaels

Virginia Commonwealth University

Follow this and additional works at: https://scholarscompass.vcu.edu/etd

Part of the Chemistry Commons

(C) The Author

\section{Downloaded from}

https://scholarscompass.vcu.edu/etd/789

This Thesis is brought to you for free and open access by the Graduate School at VCU Scholars Compass. It has been accepted for inclusion in Theses and Dissertations by an authorized administrator of VCU Scholars Compass.

For more information, please contact libcompass@vcu.edu. 


\title{
Quantitative Model for the Prediction of Hydrodynamic Size of Nonionic Reverse Micelles
}

\begin{abstract}
A thesis submitted in partial fulfillment of the requirements for the degree of Master of
\end{abstract} Science at Virginia Commonwealth University

\author{
by \\ Melissa Ann Michaels \\ B.A., Randolph-Macon Woman's College, 2003
}

Director: Dr. Everett E. Carpenter

Assistant Professor of Chemistry

Virginia Commonwealth University

Richmond, Virginia

April 2006 


\section{Table of Contents}

List of Figures $\quad$ iv

List of Tables $\quad$ vii

Abstract viii

Chapter 1: Introduction $\quad 2$

1.1 Introduction and Research Goals 3

Chapter 2: Surfactants and Micelles $\quad 11$

2.1 Introduction $\quad 12$

2.2 Driving Forces of Micellization $\quad 14$

2.3 Evidence of Micellization 18

2.4 Structure and Equilibrium 19

2.5 Types of Surfactants $\quad 22$

2.6 Choosing a Surfactant System 23

2.7 Why Nonionic Surfactants $\quad 26$

2.8 Surfactant Curvature $\quad 26$

2.9 Role of Co-surfactants $\quad 30$

2.10 Conclusions 31

$\begin{array}{ll}\text { Chapter 3: Characterization } & 33\end{array}$

3.1 Introduction $\quad 34$

3.2 Small Angle Scattering $\quad 34$

3.3 Dynamic Light Scattering 36

$\begin{array}{ll}\text { 3.3.1 DLS Fundamentals } & 37\end{array}$

3.3.2 Correlator Function $\quad 39$

3.3.3 Instrumentation $\quad 45$

3.3.4 DLS Performance 48

Chapter 4: Central Composite Design and Statistical Analysis 50

4.1 Introduction $\quad 51$

4.2 Central Composite Design 51

4.3 Phase Space $\quad 54$

4.4 Statistical Analysis $\quad 57$ 
$\begin{array}{lr}\text { Chapter 5: Results } & 60\end{array}$

5.1 Model Results 61

$\begin{array}{ll}5.2 \text { Effects of Chain Length } & 63\end{array}$

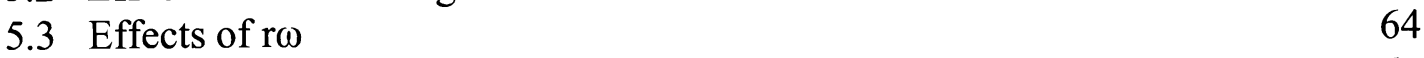

$\begin{array}{ll}5.4 \text { Effects of } \omega & 67\end{array}$

5.5 Effects of Water Concentration $\quad 69$

$\begin{array}{ll}5.6 & \text { Salt Concentration }\end{array}$

$\begin{array}{lll}5.7 & \text { Temperature } & 72\end{array}$

$\begin{array}{lll}5.8 & \text { Polar Phase Effects } & 74\end{array}$

5.9 Hydrocarbon Phase Effects 76

$\begin{array}{ll}\text { Conclusions } & 78\end{array}$

$\begin{array}{lr}\text { Appendix } & 80\end{array}$

$\begin{array}{ll}\text { References } & 82\end{array}$ 


\section{List of Figures}

\section{Chapter 1}

1.1 Chemical structures of two commonly used anionic surfactants, $\mathrm{CTAB}$ and AOT.

1.2 Chemical structure of the surfactant Nonylphenol polyethoxylate.

For the NP system, $n$ indicates the chain length, i.e. if $n=4$, then it is referred to as NP4.

\section{Chapter 2}

2.1 A pseudo phase diagram of surfactant, water and oil illustrating the regions in which micelles and reverse micelles form. The ratio of surfactant, water, and oil governs what phase the mixture will form.

2.2 Oil in water emulsion (micelle) and water in oil emulsion (reverse micelle). Transition from a bulk water core to hydrogen bonded water molecules is indicated by the color gradient.

2.3 Formation of micelles at CMC.

2.4 Important manifestation of micelle formation: abrupt changes in solution conductivity, a discontinuity in the surface tension vs. concentration curve and a sudden increase in solution turbidity.

2.5 Irregular surface of micelle compared to smooth uniform surface.

2.6 Equilibrium of surfactant monomers and reverse micelles in solution.

2.7 Chemical structure of the surfactant Nonylphenol polyethoxylate.

2.8 The critical packing parameter $\mathrm{P}_{\mathrm{c}}$ relates the head group area, the extended length and the volume of the hydrophobic part of a surfactant molecule into a dimensionless number $P_{c}=v / a_{0} l_{c}$. 


\section{Chapter 3}

3.1 The essential parts of a small angle scattering system. The drawing shows the X-ray source $T$, the sample $S$, the scattering angle $\theta$, the slits used to define the incident and scattered beams, and the detector $D$.

3.2 Speckle pattern consisting of bright light and dark areas from constructive and destructive interference, respectively.

3.3 Propagated waves from the light scattered by particles. Bright areas of light are from particles arriving at the screen with the same phase and they interfere constructively to form a bright patch. The dark areas are where the phase additions are mutually destructive and cancel each other out.

3.4 A typical correlation function of correlation against time showing the rate of decay for the correlation function is related to particle size- the rate of decay is much faster for small particles than it is for large.

3.5 Relationship of correlation function $\mathrm{C}(\tau)$ and translational diffusion coefficient (D).

3.6 A typical size distribution graph where the $\mathrm{X}$ axis shows size classes, while the $Y$ axis shows the relative intensity of the scattered light. This is therefore known as an intensity distribution.

3.7 Schematic of a typical DLS system showing its six main components:

(1) laser; (2) sample cell; (3) detector; (4) attenuator; (5) correlator; and (6) computer.

3.8 AOT/isooctane reverse micelle data obtained via DLS overlaid over Herrera's AOT/isooctane reverse micelles to verify instrument performance.

\section{Chapter 4}

4.1 Asymmetric central composite design of superimposed box and star designs ( $r, \omega,[N P])$ illustrating experiments for determination of interaction and second-order parameters.

4.2 Pseudo phase diagram of surfactant, water and oil illustrating the regions in which micelles and reverse micelles form. 


\section{Chapter 5}

5.1 Effects of $r$ on NP4/7 reverse micelle size where $\omega=6$ and $[\mathrm{NP}]=0.4$. The dotted lines represent standard error of the model predictions, and the equation for the model is represented by the solid line.

5.2 Three-dimensional cross section of the model for predicting reverse micelle size from the experimental conditions. $r$ and $\omega$ axes are surfactant to total surfactant ratio and water to surfactant molar ratio, respectively. This cross section is for a constant $[\mathrm{NP}]$ of $0.4 \mathrm{M}$.

5.3 Effects of $\omega$ on NP4/7 reverse micelle size where $r=0.25$ and $[\mathrm{NP}]=0.4$. The dotted lines represent standard error of the model predictions, and the equation for the model is represented by the solid line.

5.4 Effect of $[\mathrm{NP}]$ on NP4/7 reverse micelle size where $r=0.25$ and $\omega=6$. The dotted lines represent standard error of the model prediction, and the equation for the model is represented by the solid line.

5.5 Three-dimensional cross section of the model for predicting reverse micelle size from the experimental conditions. [NP] and $\omega$ axes are surfactant concentration and water to surfactant molar ratio, respectively. This cross section is for a constant $r$ of $0.25 \mathrm{M}$.

5.6 Reverse micelle size dependence on $\mathrm{NaCl}$ concentration. All points fall within standard deviations of each other indicating no dependence.

5.7 Temperature-dependence of NP4/7 reverse micelle size corrected for viscosity where $r=0.25, \omega=6$, and $[\mathrm{NP}]=0.4$. 


\section{List of Tables}

\section{Chapter 1}

None

\section{Chapter 2}

2.1 HLB ranges and their general areas of application.

\section{Chapter 3}

None

\section{Chapter 4}

4.1 Experimental conditions for box and star CCD containing 23 points13 star, 8 box, as well as 2 additional center replicates. $\omega$ varied from 2 - 8; [NP] varied from $0.3 \mathrm{M}-0.5 \mathrm{M}$; and $\mathrm{r}$ varied from $0.15-0.30$. Predicted sizes were obtained from equation 4.2 .

4.2 Original star design centered at $[\mathrm{NP}]=0.3 \mathrm{M}$. Additional star points used to calculate the model for NP4 and NP7 (equation 4.2).

\section{Chapter 5}

5.1 Model coefficients and results from backward elimination method in SPSS demonstrating $p$-values and $95 \%$ confidence intervals.

5.2 Validation experiments to test the model for NP4/NP7 reverse micelle size. 


\begin{abstract}
QUANTITATIVE MODEL FOR THE PREDICTION OF HYDRODYNAMIC SIZE OF NONIONIC REVERSE MICELLES
\end{abstract}

\author{
By Melissa A. Michaels, M.S. \\ A thesis submitted in partial fulfillment of the requirements for the degree of Master of \\ Science at Virginia Commonwealth University. \\ Virginia Commonwealth University, 2006 \\ Director: Dr. Everett E. Carpenter \\ Assistant Professor of Chemistry
}

The sizes of nonionic reverse micelles were investigated as a function of the molecular structure of the surfactant, the type of oil, the total concentration of surfactant [NP], the ratio of NP4 to total surfactant (r), the water to surfactant molar ratio $(\omega)$, temperature, salt concentration, and polar phase. The basis of our investigation was nonylphenol polyethoxylates-NP4 and NP7. Micelle sizes were determined using dynamic light scattering (DLS). A central composite experimental design was used to quantitatively model reverse micelle size as a function of $\omega,[\mathrm{NP}]$, and $\mathrm{r}$. The model has demonstrated the capability of predicting the mean diameter of micelles from 4 to $13 \mathrm{~nm}$ with a precision of \pm $2 \mathrm{~nm}$ as measured by DLS. This quantitative correlation between the size of reverse micelles and the synthetic variables provides the foundation for choosing experimental conditions to control reverse micelle size. 


\section{QUANTITATIVE MODEL FOR THE PREDICTION OF HYDRODYNAMIC SIZE OF NONIONIC REVERSE MICELLES}




\section{Chapter 1: Introduction}

If a bear in Yosemite and one in Alaska fall into water, which one would dissolve faster?

The bear in Alaska because it's polar. 


\subsection{Introduction and Research Goals}

There has been a strong focus on developing procedures for controlling the particle size of synthetic nanomaterials ever since the discovery of the advantageous properties of ultra small particles in the early $1980 \mathrm{~s} .{ }^{1-3}$ These small groups of atoms go by many names —nanoclusters, nanocrystals, and quantum dots, but are commonly called nanoparticles. ${ }^{4,5}$ Because particle size has a direct influence on the material's physical properties such as magnetic moment or optical spectrum, control of the size is a general goal of nanoparticle research. ${ }^{6}$ Size control is usually achieved during synthesis by varying the experimental conditions until the desired size is obtained. Physically manipulating the size and shape of the particles, which is a common procedure in processing bulk product, is not practical because of the difficulty in manipulating material on the nanoscale. ${ }^{7}$ To provide control in producing a desired nanoparticle size, it is ideal to develop a model for the relationship between the synthetic parameters and the resultant nanoparticle size. Moreover, nanoparticles with wide size distributions have property distributions that are also broad. ${ }^{6}$ For this reason, research has focused on finding ways to synthesize uniform-sized nanoparticles because they will possess very specific optical, electrical, and magnetic properties.

Current research shows that nanoparticles exhibit many fascinating sizedependent properties. It would be advantageous to explore the size-dependent effects if the size of the nanoparticle can be reliably tailored. Therefore, depending on the intended function of the material, most manufacturers are interested in the ability to control 
particle size, particle shape, size distribution, particle composition, and the degree of particle agglomeration.

A variety of chemical techniques have been developed for the synthesis of nanoparticles in recent years. ${ }^{8-14}$ There are two general ways available to produce nanomaterials. The first way is to start with a bulk material and then break it into smaller pieces using mechanical, chemical or other forms of energy (a top-down or physical method). An opposite approach is to synthesize the material from atomic or molecular species via chemical reactions, allowing for the precursor particles to grow in size (a bottom-up or chemical method). Both approaches can be done in either gas, liquid, or solid states, as well as in supercritical fluids or in vacuum.

A wide range of nanoparticle syntheses have been developed via physical methods that include vapor condensation, spray pyrolysis, flame decomposition, and ball milling. ${ }^{15-18}$ As mentioned earlier, these methods produce nanomaterials by reducing bulk material; however they are limited in their ability to control many properties that are desired in nanoparticles. Syntheses involving a vapor phase such as in vapor condensation, or mechanical abrasion such as in ball-milling, have been extensively investigated, however results have shown that particles in a gas phase will always agglomerate and mechanical abrasion may lead to polydisperse size distributions. These are key disadvantages since many potential applications require dispersed spherical nanoparticles of uniform size.

Chemical methods are based upon solution phase routes to nanoparticle synthesis and include the use of soluble surfactant molecules, ligands and/or polymers, for assisting 
the growth of nanoparticles in the solution phase (microemulsion technique). These chemical methods are much more suitable for tailoring small and uniform nanoparticles than the physical method since physical methods tends to impart physical stresses in the material which can require further processing and result in wider size distributions; however, it is generally easier to produce large volumes of product via a physical method. ${ }^{19}$

In contrast to physically dividing bulk materials, chemical methods, such as those based on sol-gel, hydrothermal processing, reduction methods, and micelles, grow particles from atomic or molecular species. These methods typically involve some form of ligand to assist particle growth and are performed in solution at low temperatures providing kinetically stable particles. A problem with some of these techniques, such as aqueous co-precipitation and borohydride reduction, is their lack of control over particle growth which leads to broad particle size distributions. ${ }^{20-25}$ However, methods such as reverse micelle syntheses have demonstrated considerable control over both the size and size distribution of the resultant nanomaterial product. ${ }^{26}$ Reactions such as coprecipitation and reduction for nanoparticle synthesis can be done within a micelle system. Micelles provide a mechanism for controlling the particle size while providing a narrow $8 \%$ standard deviation of size distribution. ${ }^{27,28}$ The ability of this reaction system to control the size and size distribution makes it attractive for the synthesis of nanoparticles.

Reverse micelles occur in a small region of phase space formed from a mixture of surfactant, oil, and water, where the oil phase is continuous. Reactions such as 
oxidations, reductions, and precipitations can be carried out in the aqueous pools formed by water surrounded by the surfactant and isolated from the oil phase. Investigators have suggested that reverse micelles act as nanoreactors where the size of the water pool can be controlled by various parameters such as ionic strength, surfactant concentration, and particularly the water to surfactant molar ratio $(\omega) .{ }^{29-33}$ Previous work has shown that the size of micelle water pools could also be affected by the molecular structure of the surfactant. $^{34}$

A general methodology for synthesizing nanoparticles employing reverse micelles can be developed using one of two similar routes. ${ }^{35}$ The first case involves dispersing the reactants in separate reverse micelle solutions and then mixing the two solutions. In this process the reverse micelles coalesce and exchange of the materials within the water droplets occurs. This allows the reaction to take place in the water pools and nanoparticles are subsequently formed within the reversed micelles. The second case involves mixing one reactant that is solubilized in the reversed micelles with another reactant that is dissolved in water, i.e. aqueous phase exchange. The first method is more often used for nanoparticle synthesis within reverse micelles, although there has been syntheses reported using the latter method. ${ }^{34,36}$

Different types of surfactants can produce micelles. Cationic surfactants, such as cetyltrimethylammonium bromide (CTAB) as well as anionic surfactants such as sodium bis(2-ethylhexyl) sulfosuccinate, more commonly known as AOT (see Figure 1.1), have been used extensively for research into the fundamental physical chemistry of surface activity. 


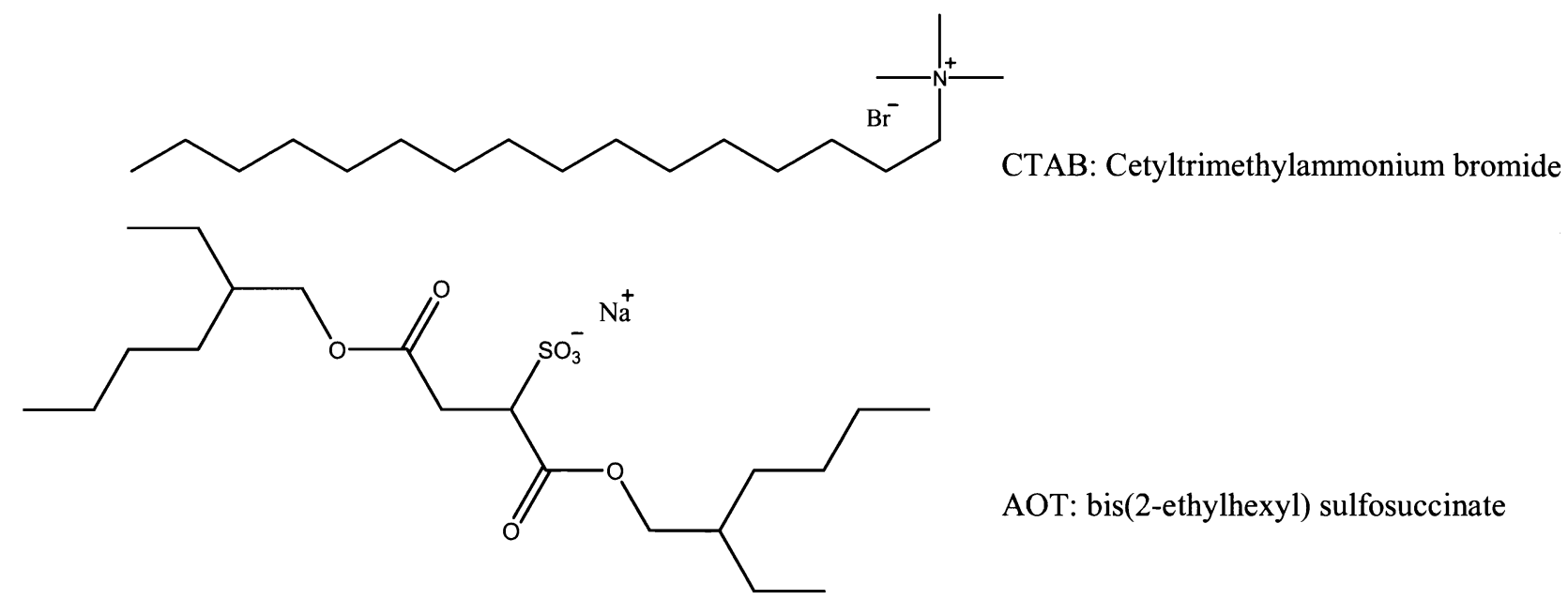

Figure 1.1: Two commonly used cationic and anionic surfactants for reverse micelles, CTAB and AOT respectively.

By far, the largest class of surfactants in general use today is anionic. Because of their utility and ease of manufacture, the sulfonate surfactants have been the subject of extensive research efforts. Almost every conceivable class of organic compound has been subjected to the action of sulfonating reagents in hopes of finding a "supersurfactant" with the surface activity to dominate a large portion of the market. ${ }^{37}$

AOT is one of the most widely studied and well documented anionic surfactants in the field of reverse micelles. AOT has a small polar head group with large, branching tail groups. This makes for a three dimensional "wedge" shape, which favors spherical micellar arrangement. ${ }^{36}$ Isooctane is typically used with AOT because it has a structure similar to the tail structure of AOT and thus has the best penetration into AOT tails. ${ }^{38}$ In an aqueous solution, AOT spontaneously forms a micellar structure; however, in an organic solvent, a reverse micelle is formed. 
AOT is particularly well suited for forming reverse micelles due to its ability to form them in hydrocarbon oils at different concentrations as well as its ability to solubilize a large amount of water with water to surfactant molar ratio $(\omega)$ up to 60 and offers a wider range of nanoparticle sizes..$^{39-44}$ However, unfavorable interactions between ionic reactants and surfactant head groups have triggered interest in using nonionic surfactants for reverse micelles, specifically in applications such as nanoparticle growth. Although the two surfactant classes discussed so far can be characterized by the existence of a net electrical charge, such a situation is not a fundamental requirement for the existence of surface activity (i.e., interfacial tension) in water. Nonionic surfactants have many unique properties that are superior to those of ionic surfactants with comparable hydrophobic groups, such as remarkably low critical micelle concentrations, ${ }^{26,45,46}$ high efficiency in reducing surface tension, ${ }^{46,47}$ and better solubilizing properties, which make them potentially useful in a wide variety of industrial applications. Some of the most important advantages can include a significantly lower sensitivity to the presence of electrolytes in the system, a lessened effect of solution $\mathrm{pH}^{37}$ and the synthetic flexibility of being able to design the required degree of solubility into the molecule by careful control of the size of the hydrophilic group. In addition, surfactant systems consisting of cationic or anionic hydrophilic groups contain external layers of water (as opposed to the bulk) within the micelle that are bound more tightly to the water interface when compared to nonionic systems. ${ }^{37}$ This effectively reduces the size of the reaction volume. Although these effects are not characterized well enough to perform a direct evaluation, they are present and can produce noticeable differences in 
comparing products from different reverse micelle systems. ${ }^{36}$ Therefore, nonionic surfactants are of particular interest for reverse micelle systems and will be discussed in further detail.

The nonionic surfactant nonylphenol polyethoxylate (NP), sold by Rhodia as the commercial surfactant IGEPAL ${ }^{\circledR}$ (Figure 1.2), has been reported to form reverse micelles in hydrocarbon oils. ${ }^{33}$

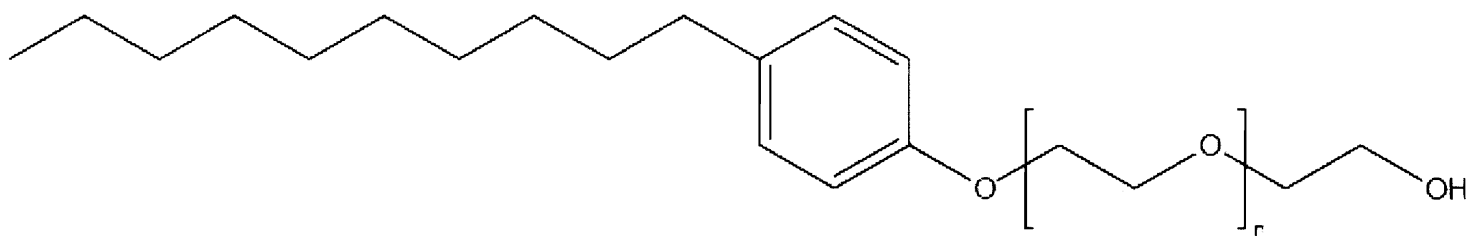

Figure 1.2: Chemical structure of the surfactant Nonylphenol polyethoxylate. For the NP system, $n$ indicates the chain length, i.e. if $n=4$, then it is referred to as NP4.

IGEPAL ${ }^{\circledR}$, having no charged counter ion, binds less tightly to nanoparticles compared to AOT therefore making the surfactant easier to remove after synthesis. ${ }^{36}$ This allows a faster cleanup procedure that uses less solvent. For these reasons, NP reverse micelles are of particular interest for nanoparticle synthesis.

As mentioned earlier, reverse micelle systems have demonstrated considerable control over both the size and size distribution of the resultant nanomaterial. Since reverse micelles act as nanoreactors where the size of the water pool is linked to the size of the nanoparticle synthesized within it, in theory, a larger sized micelle should produce a larger sized nanoparticle. Therefore, in order to synthesize nanoparticles with better size control, the objectives of this work are: (1) to determine the main parameters affecting the size of NP reverse micelles; (2) to explain how the size of NP reverse 
micelles are controlled by these parameters; and (3) to establish a quantitative model that correlates reverse micelle size with synthetic conditions by using a statistical experimental design method. The effects of $\omega=\left[\mathrm{H}_{2} \mathrm{O}\right] /[\mathrm{NP}]$, surfactant molecular structure, $r=\left[\mathrm{NP}_{4}\right] /[\mathrm{NP}]_{\mathrm{T}}$, total surfactant concentration $[\mathrm{NP}]_{\mathrm{T}}$, temperature, salt concentration, polar phase, and oil phase on the formation and size of reverse micelles were the parameters investigated in this work. Since the reverse micelle technique is the method employed in the current study, a more extensive description of this technique and its applicability to nanoparticle synthesis will be provided in Chapter 2. Dynamic light scattering (DLS) was used to characterize the influence of investigated parameters on reverse micelle size and is discussed in Chapter 3. The statistical method of central composite design (CCD) allows the relationship between parameters and reverse micelle size obtained from DLS to be modeled and is presented in Chapter 4. In addition, the analysis of the synthetic variables to produce a model that predicts reverse micelle size is discussed in Chapter 5. 
Chapter 2: Surfactants and Micelles

A small piece of sodium that lived in a test tube fell in love with a Bunsen burner.

"Oh Bunsen, my flame... I melt whenever I see you...," the sodium pined.

"It's just a phase you're going through", replied the Bunsen burner. 


\subsection{Introduction}

Before detailing the model for the relationship between synthetic conditions and reverse micelle size, a description of the reverse micelle technique and how and why reverse micelles form will be discussed. In addition, the nature of surfactants will be explained. As previously mentioned, reverse micelles occur in a small region of phase space formed from a mixture of surfactant, oil, and water, where the oil phase is continuous (see Figure 2.1). The region of space lying at the boundary between two immiscible phases, such as oil and water, is generally referred to as the "interface" and represents a transition region in which the chemical and physical characteristics of one bulk phase undergo an abrupt (on the macroscopic scale) change to those of the adjacent one. On a microscopic scale, however, that change must occur over the distance of at least one, but more often several, molecular distances.

An emulsion is formed when water and oil are mixed together to produce a stable suspension of one within the other. ${ }^{48}$ A surfactant molecule has an affinity for both solvents and therefore generally assists this process by serving as the boundary between the two liquid phases.

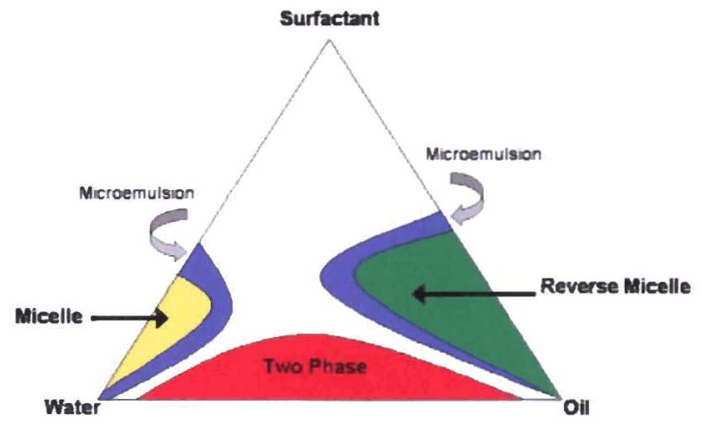

Figure 2.1: A pseudo phase diagram of surfactant, water and oil illustrating the regions in which micelles and reverse micelles form. The ratio of surfactant, water, and oil governs what phase the mixture will form. 
An emulsion can be either oil droplets suspended in water, an oil in water $(\mathrm{O} / \mathrm{W})$ emulsion, or water suspended in a continuous oil phase, a water in oil (W/O) emulsion (Figure 2.2). When micelles form in water, the surfactant tails form a core that is like an oil droplet, and their polar heads form an outer shell that maintains favorable contact with water. When surfactants assemble in oil, the aggregate is referred to as a reverse micelle. In a reverse micelle, the heads are in the core and the tails maintain favorable contact with the oil phase.
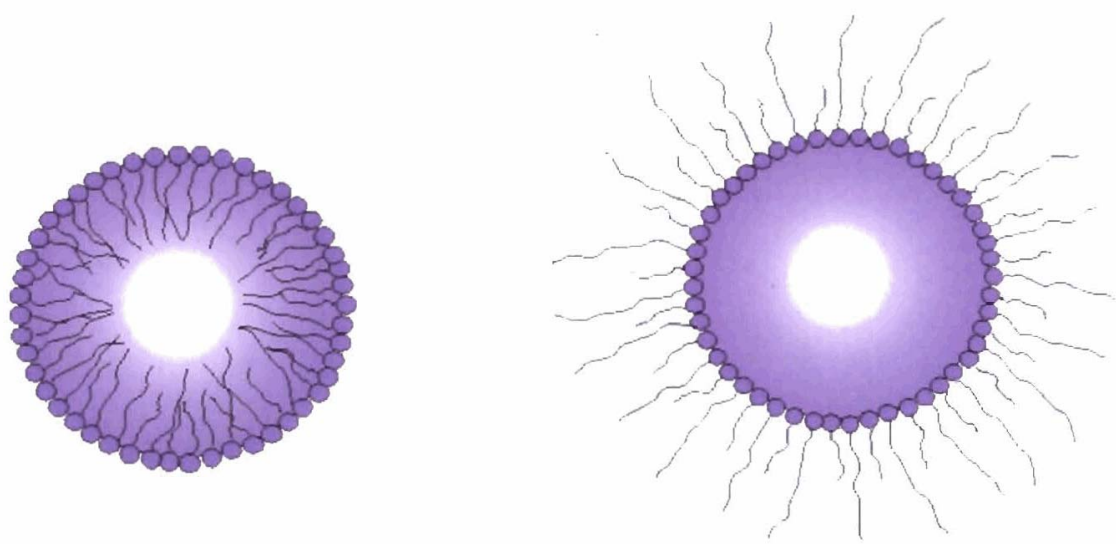

Figure 2.2: Oil in water emulsion (micelle) and a water in oil emulsion (reverse micelle). Transition from a bulk water core to hydrogen bonded water molecules is indicated by the color gradient.

It is reverse micelles, i.e., W/O emulsions, that are useful systems for growing nanoparticles based on aqueous, room temperature reactions. As previously discussed, reactions evolve within the water pools which then restrict the growth of the particles and limit their size. Studies of micelle systems have demonstrated that the water pools are uniform in size, thereby providing an environment in which to produce uniform nanoparticles of a given size. ${ }^{7,28,33,36}$ Since the selection of surfactants and relative 
amounts of the two phases determine the class of emulsion, it is important to elucidate how and why micelles form.

\subsection{Driving Forces of Micellization}

A surfactant molecule has two parts: a nonpolar hydrocarbon chain (designated as hydrophobic or lipophilic) and a polar head group (hydrophilic or lipophobic). These two structures in the same molecule form a special amphiphilic molecular structure that possesses distinctive surface activity (discussed further in Section 2.3) and is known as a surfactant. Such a molecular structure poses a double property: one part can dissolve in water and the other can dissolve in oil-like materials.

The amphiphilic nature of surfactant molecules causes them to possess many properties that appear to be contradictory. Because of their chemical composition they have something of a "love/hate" relationship with most solvents, which results in a constant tug-of-war between forces to achieve a comfortable accommodation with a given environment. As previously mentioned, the presence of an interface induces an imbalance of forces that alters the energetic situation of molecules at or near that interface usually giving molecules in that region a higher net energy than those in the bulk. The drive to lower the energy of the system resulting from the presence of the interface is one factor that results in preferential adsorption of surfactants to interfaces. Therefore, in their drive to satisfy the need to minimize energetically unfavorable interactions with their environment, surfactants will spend most of their time at interfaces or with others of their kind. ${ }^{37}$ The nature of surfactant molecules, having both 
hydrophilic and hydrophobic groups, is responsible for their tendency to concentrate at interfaces and thereby reduce the free energy of the system in which they interact. The "dislike" for water of the hydrophobic portion of a surfactant molecule is not an actual repulsive interaction; neither does there exist a strong attraction between the hydrophilic chains of surfactant monomers since their interactions are polar, and therefore, relatively small. Rather, there exists a polar association among the polar chains.

Because of its chemical nature, water possesses a very strong cohesive force, which results in many of its unusual properties. ${ }^{49}$ When a surfactant is introduced into water, a distortion of the water structure occurs (i.e., hydrogen bonds) to accommodate the solute molecules, which increases the free energy of the system. The physical result of such an energy increase is a tendency for the solute molecules to adsorb at solution interfaces where preferred molecular orientations may reduce the free energy of solution; however, when all interfaces are saturated, the overall energy reduction may continue through other mechanisms. The physical manifestation of one such mechanism is the formation of molecular aggregates or micelles that remain in solution (as opposed to precipitating out) as thermodynamically stable, dispersed species with properties distinct from those of the monomeric solution (discussed shortly).

The traditional picture of micelle formation thermodynamics is based on the Gibbs-Helmholtz equation:

$$
\Delta \mathrm{G}_{\mathrm{m}}=\Delta \mathrm{H}_{\mathrm{m}}-\mathrm{T} \Delta \mathrm{S}_{\mathrm{m}}
$$

The formation of micelles has been found to result in a large, negative change in Gibbs energy, i.e., the aggregation process is thermodynamically favored and spontaneous. ${ }^{50,51}$ 
When surfactant molecules are deposited on the interface, reducing the surface tension, the free energy is lowered. Also, the water molecules surrounding free surfactant molecules form "extra" hydrogen bonds (in comparison to bulk water) to make a "shell" in which the hydrophobic part of the surfactant molecule is enclosed. When these hydrophobic parts are expelled from the surrounding water (by forming aggregates), the disruption of this ordered hydrogen bonded structure increases the entropy of the system which in turn decreases the free energy. The removal of the hydrophilic group from the oil through micelle formation is associated with a large increase in entropy and an enthalpy of approximately zero, resulting in a large and negative increase in the free energy of the system. ${ }^{49}$ The situation then becomes a tug-of-war between the opposing free energy considerations. At elevated temperatures, however, the increase in entropy does not account for the large negative change in Gibbs energy. ${ }^{52,53}$ For many hydrophobic compounds, entropy change approaches zero at high temperatures and becomes negative at temperatures above $130^{\circ} \mathrm{C}$. In this case, the major contribution for Gibbs energy comes from enthalpy, which becomes large and negative in order to compensate for the changes in entropy. ${ }^{53}$ In other words, micelle formation at high temperatures is thought to be "enthalpy-driven," whereas at room temperature, the gain in entropy is the major factor leading to the negative change in Gibbs energy. Therefore, the occurrence of micellization in a given surfactant system, and the concentration at which micelle formation occurs (critical micelle concentration, or CMC) is determined by the relative balance of the forces favoring and impeding the molecular aggregation process. Figure 2.3 illustrates this threshold concentration at which micelles begin to 
form, i.e., the CMC. Once a sufficient amount of surfactant has been added to the water/oil solution (i.e., the $\mathrm{CMC}$ has been reached), aggregations of surfactant monomers, i.e., micelles, will form.

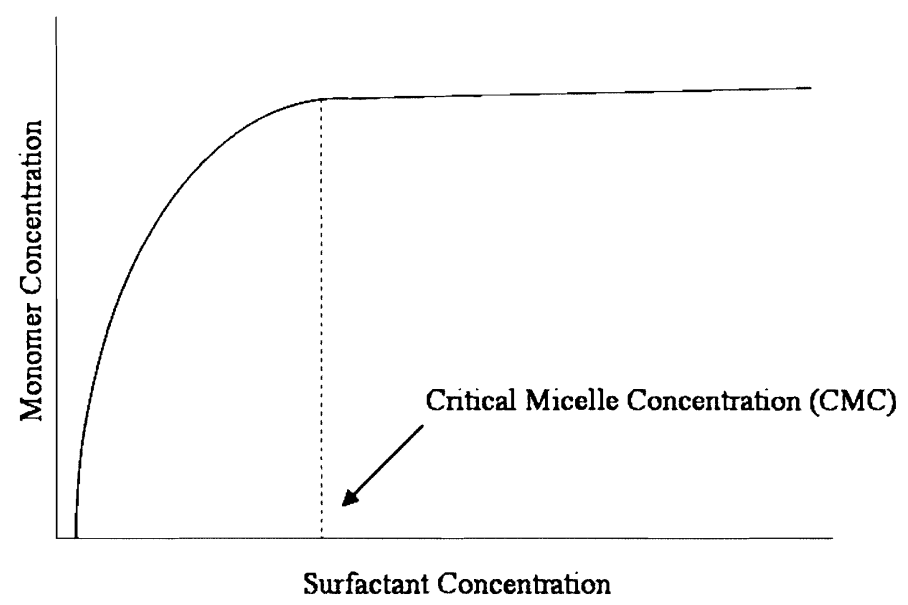

Figure 2.3: Formation of micelles at CMC.

Beyond the CMC, any surfactant added to the water/oil system will not increase the number of free monomers in solution, but rather will contribute to the formation of additional micelles. At low concentrations, surfactants will favor arrangement on the surface. As the solution becomes crowded with surfactant, more molecules will arrange into micelles; therefore, a lower CMC indicates that micelles are formed at lower concentrations of surfactant in solution.

Since the magnitudes of the opposing forces are determined by the chemical compositions of the solute molecules, where all other aspects (temperature, pressure, solvent, etc.) are held constant, it is the chemical constitution of the surfactants and the system that ultimately controls micellization. 


\subsection{Evidence of Micellization}

Early in the study of the solution properties of surfactants, it became obvious that the bulk solution properties were unusual and could change dramatically over very small concentration ranges. The measurement of bulk solution properties such as surface tension, electrical conductivity, or light scattering as a function of surfactant concentration produces curves that normally exhibit sharp discontinuities (Figure 2.4).

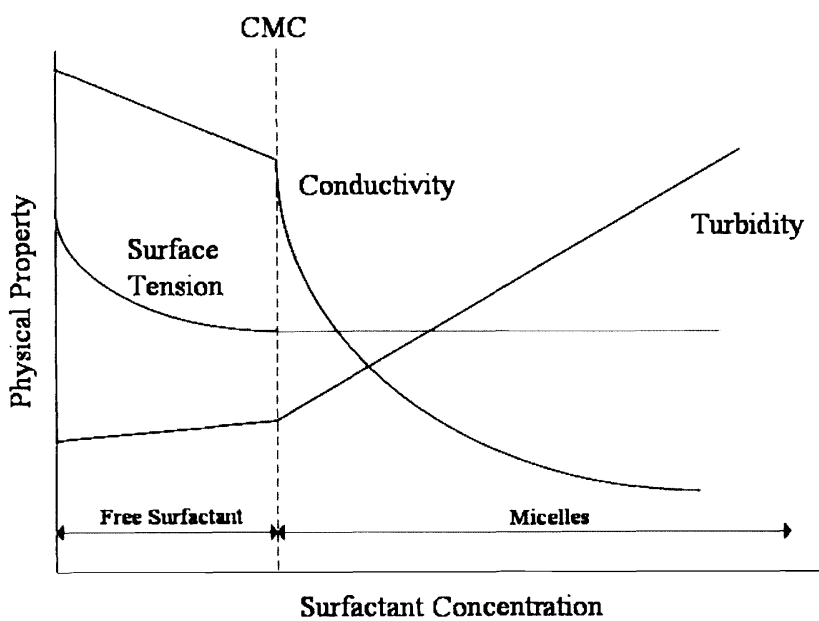

Figure 2.4: Important manifestation of micelle formation: abrupt changes in solution conductivity, a discontinuity in the surface tension vs. concentration curve and a sudden increase in solution turbidity.

The sudden change in a measured property indicates a significant change in the nature of the solute species. It has been suggested that in the case of the measurement of conductivity, the break may be associated with an increase in the mass per unit charge of the conducting species. ${ }^{49}$ For light scattering, the change in solution turbidity indicates the appearance of a scattering species of significantly greater size than the monomeric surfactant molecule. In addition, above the $\mathrm{CMC}$, the discontinuity of oil and water phases no longer exists, i.e., the evolution of a single continuous oil phase instigates the 
change in solution turbidity. These, as well as many other types of measurements, ${ }^{54}$ serve as evidence for the formation of micelles in solutions of surfactants at relatively well defined concentrations.

\subsection{Structure and Equilibrium}

As discussed earlier, micelle size and shape are governed by geometric and energetic considerations. Although micelles have various shapes (cylindrical, lamellar, disk-like), they are often spherical in shape and typically contain 50-200 surfactant monomers. ${ }^{37}$ In addition, since it is reverse micelles that are useful reaction vessels for nanoparticle synthesis, further mention of micelles in this work will refer specifically to spherical reverse micelles.

While the formation of many small micelles is kinetically favored (i.e., they nucleate more easily), large micelles are thermodynamically favored. Thus, from a standpoint of kinetics, it is easier to nucleate many small micelles. However, small micelles have a larger surface area (of surfactant) to volume (of water) ratio than large micelles. Large micelles, with their greater volume to surface area ratio, represent a lower energy state. Thus, many small micelles will attain a lower energy state if transformed into large micelles. For these reasons, the molar ratio of water to surfactant ( $\omega)$ could play an important role in determining the size of reverse micelles and was therefore investigated.

It is unreasonable to assume that surfactant molecules pack into a micelle in such an orderly manner as to produce a smooth, perfectly uniform surface structure. If it were 
possible to photograph a micelle with ultra-high-speed film, freezing the motion of the molecules, the picture would probably show an irregular molecular cluster more closely resembling a sweetgum seed than a golf ball (Figure 2.4).
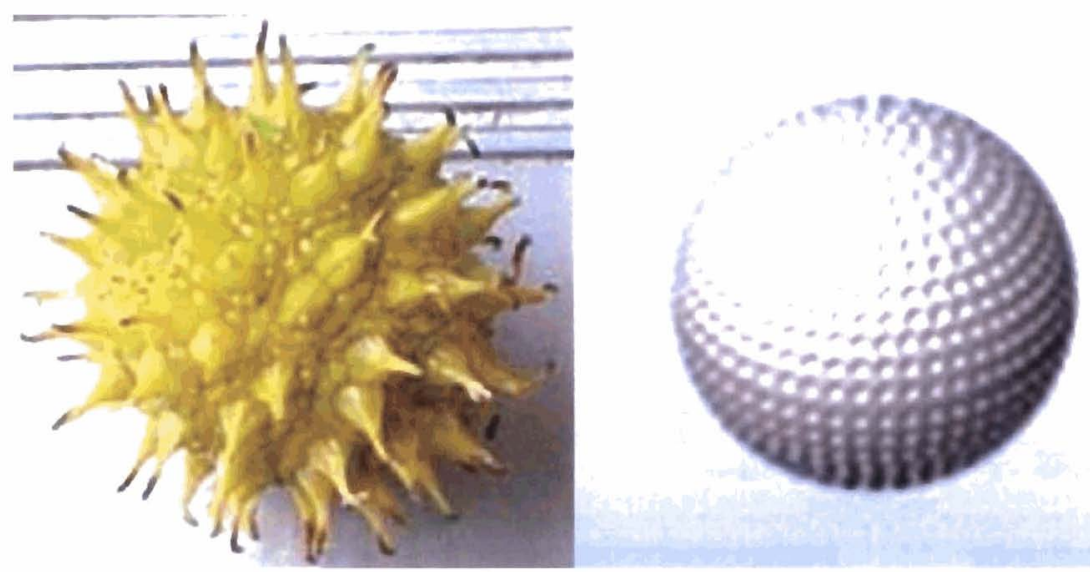

Figure 2.5: Irregular surface of micelle compared to smooth uniform surface.

Experimental results show that micelles are in dynamic equilibrium with individual surfactant molecule monomers that are constantly being exchanged between the bulk and the micelles. ${ }^{37}$ Additionally, micelles themselves are continuously dissoluting and reforming. ${ }^{55}$ The kinetics of micellization has been studied by various techniques such as stopped flow, temperature jump, pressure jump, and ultrasonic absorption. ${ }^{55}$ There are two relaxation processes involved in micellar solutions. The first one is the fast relaxation process with relaxation time $\tau_{1}$ (generally on the order of microseconds ${ }^{55}$ ), which is associated with the fast exchange of monomers between micelles and the surrounding bulk phase (Figure 2.5). The second relaxation time $\tau_{2}$ (usually on the order of milliseconds ${ }^{55}$ ) is attributed to the micelle formation and dissociation process. These two relaxation times are used to calculate two important 
parameters of a micelle solution: (1) the residence time of a surfactant molecule in a micelle and (2) the average lifetime or stability of micelles. The stability of micelles is important since even small changes in the stability can have an effect on the interfacial and bulk properties of the solutions, e.g., surface tension and viscosity.

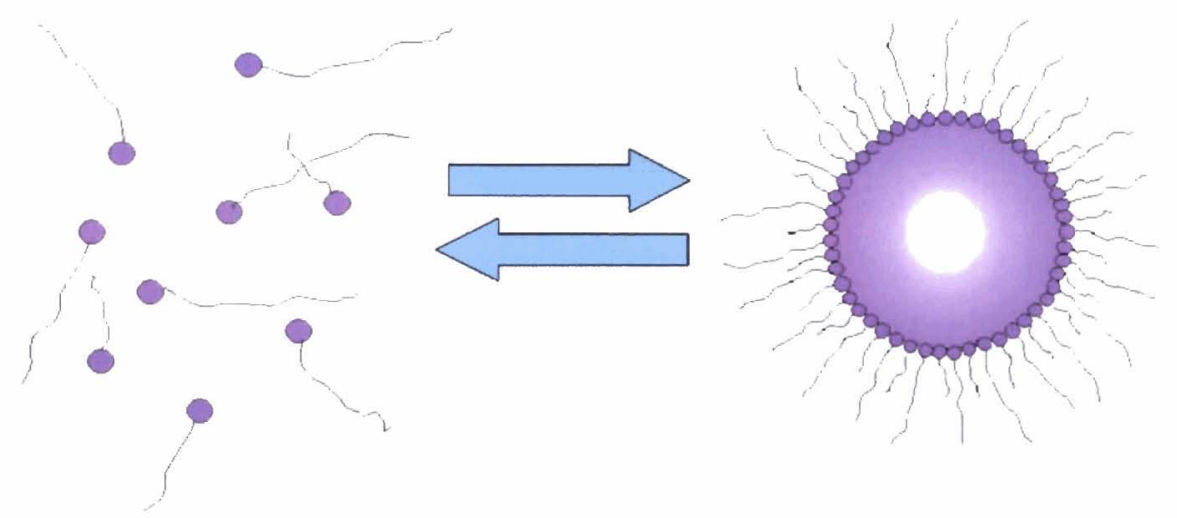

Figure 2.6: Equilibrium of surfactant monomers and reverse micelles in solution.

This chemical equilibrium between surfactant monomers and micelles is known as the mass action model and is accepted as useful for understanding the energetics of the process of micellization; this model can be expressed in the following form:

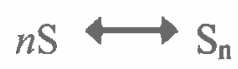

where $\mathrm{n}$ is the number of molecules of surfactant, $\mathrm{S}$, associating to form the micelle, i.e., the aggregation number. Equilibrium between monomeric surfactant and micelles is assumed with a corresponding equilibrium constant, $\mathrm{K}_{\mathrm{m}}$, given by

$$
K_{m}=\frac{[\text { micelles }]}{[\text { monomers }]^{n}}=\frac{\left[S_{n}\right]}{[S]^{n}}
$$


From Eq. 2.3, the standard free energy of micellization per mole of micelles is given by

$$
\Delta G_{m}^{\circ}=-R T \ln K_{m}=-R T \ln S_{n}+n R T \ln S
$$

while the standard free energy change per mole of surfactant is

$$
\frac{\Delta G_{m}^{\circ}}{n}=-\frac{R T}{n} \ln S_{n}+R T \ln S
$$

Assuming $n$ is large $(\sim 100)$ the first term on the right side of Eq. 2.5 can be neglected, and an approximate expression for the free energy of micellization per mole of neutral surfactant becomes

$$
\Delta G_{M, m}^{\circ} \approx R T \ln (C M C)
$$

In the case of ionic surfactants, the expression for the free energy of micellization is different since the presence of the counter ion and its degree of association with the monomer and micelle must be considered.

\subsection{Types of Surfactants}

Recall the two portions of a surfactant molecule are a hydrophobic group and hydrophilic group. Hydrophobic groups may be large, straight or branched chain hydrocarbons, cyclic hydrocarbons, aromatic hydrocarbons and/or combinations of them. Surfactants can be classified according to their physical properties or functionalities. The following is the most common classification and is based on the nature of the hydrophilic group. 
Anionic

* surface-active portion exhibits a negative charge

* tend to be good solubilizers

* examples include sulfonic acid salts, phosphoric acid esters, alcohol sulfates, and carboxylic acid salts ${ }^{37}$

Cationic

* surface-active portion exhibits a positive charge

* examples include polyamines and their salts, quaternary ammonium salts, and amine oxides ${ }^{37}$

* potential problems since they tend to absorb to anionic surfaces

Nonionic

* surface-active portion exhibits no charge

* examples include polyoxyethylenated alkylphenols, alcohol ethoxylates, alkylphenol ethoxylates, and alkanolamides ${ }^{37}$

* tend to be good solubilizers and are usually easily blended with other types of surfactants (i.e., used as co-surfactants)

* relatively insensitive to the presence of salts in solution compared to anionic

Zwitterionic

* surface-active portion contains both negative and positive functional groups

* examples include imidazoline derivatives, betaines, amino acid derivatives and lecithins ${ }^{37}$

* represent only a small portion of surfactant production $(\sim 1 \%)$

* significantly increasing interest, particularly since their dual nature reduces or eliminates the possibility of undesirable polymer-surfactant interactions

\subsection{Choosing a Surfactant System}

For all classes of surfactant, the hydrophilic end is strongly attracted to water molecules. As a result, the surfactant molecules align at the surface and internally so that 
the hydrophilic end is toward the water and the hydrophobic portion is squeezed away from the water and toward the oil, forming a micelle. Because of this characteristic behavior of surfactants to orient at surfaces and to form micelles, their applicability varies with the phase to be encapsulated and each surfactant excels in certain functions and has others in which it is deficient.

Foaming agents, emulsifiers, and dispersants are surfactants which suspend, respectively, a gas, an immiscible liquid, or a solid in water or some other liquid. ${ }^{55}$ Although there is similarity in these functions, in practice, the surfactants required to perform these functions differ widely. In emulsification, for example - the selection of surfactant will depend on the materials to be used and the properties desired in the end product. The type of surfactant behavior, whether acting as an emulsifier or dispersant or otherwise, depends on the structural groups on the molecule. The hydrophile-lipophile balance (HLB) number helps define the function that a molecular group will perform.

It has been a long-term goal of surfactant chemists to devise a quantitative way of correlating the chemical structure of surfactant molecules with their surface activity to facilitate the choice of material for a particular use. The first successful attempt to correlate surfactant structures with their effectiveness as emulsifiers was the hydrophilelipophile balance system developed by Griffin. ${ }^{56}$ In this system, Griffin proposed to calculate the HLB of a surfactant from its chemical structure and to match that number with the HLB of the oil phase. The system employs certain empirical formulas to calculate the HLB number within a range of 0 to 20. Hydrophilic surfactants that possess high water solubility and generally act as good solubilizing agents, detergents, and 
stabilizers for $\mathrm{O} / \mathrm{W}$ emulsions lie at the high end of the scale; at the low end are surfactants with low water solubility, which act as solubilizers of water in oils and are good W/O emulsion stabilizers. The effectiveness of a given surfactant in stabilizing a particular emulsion system depends on the balance between the HLBs of the surfactant and the oil phase involved. Although, the HLB system proposed by Griffin has been useful in most general applications by guiding the chemist to a choice of surfactant most suited to individual needs, others have suggested HLB numbers could be calculated based upon polar and nonpolar group contributions. ${ }^{57}$ Table 2.1 lists the ranges of HLB numbers that have proved most useful for various applications. ${ }^{58}$

Table 2.1: HLB ranges and their general areas of application.

\begin{tabular}{|c|c|}
\hline Range & Application \\
\hline $3-10$ & W/O emulsions \\
\hline $7-11$ & Wetting \\
\hline $11-18$ & O/W emulsions \\
\hline $3-15$ & Detergency \\
\hline $15-18$ & Solubilization \\
\hline
\end{tabular}

The ranges in which surfactants of various HLBs can be employed are broad. Specific requirements for many systems have been tabulated by Becher as well as many others. ${ }^{59}$ While such tabulations can be useful, many surfactants may possess the same HLB, yet subtle differences in their chemical structures or physical chemistry may result in significant differences in performance. Although HLB numbers were not investigated to choose a surfactant system in this work, it is important to note that potential surfactant systems for our purposes are limited to those that have low HLBs since it is W/O emulsions that are useful for nanoparticle synthesis. 


\subsection{Nonionic Surfactants}

Of all the nonionic surfactant classes available, the polyoxyethylenes (POE) are easily the most numerous and most important. These materials have the general formula $\mathrm{RX}\left(\mathrm{CH}_{2} \mathrm{CH}_{2} \mathrm{O}\right)_{n} \mathrm{CH}_{2} \mathrm{CH}_{2} \mathrm{OH}$ where $\mathrm{R}$ is a typical surfactant hydrophobic group, and $\mathrm{X}$ is $\mathrm{O}, \mathrm{N}$, or another functionality capable of linking the polyoxyethylene chain to the hydrophobe. In most cases, $n$, the average number of oxyethylene units in the hydrophilic group, must be greater than 4 or 5 to impart sufficient water solubility to make the surfactant useful. ${ }^{37}$

The nonionic surfactant nonylphenol polyethoxylate (NP), sold by Rhodia as the commercial surfactant IGEPAL $\AA$, has been reported to form reverse micelles in hydrocarbon oils. ${ }^{33}$ This class of surfactants is based on NP incorporating a chain of ether linkages to produce a polar head group where the number following the NP is the number of ether linkages (see Figure 2.7).

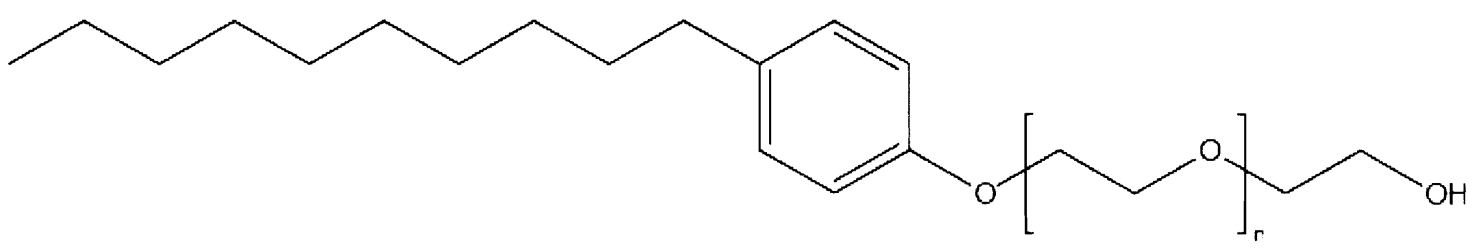

Figure 2.7: Chemical structure of the surfactant Nonylphenol polyethoxylate. For the NP system, $n$ indicates the chain length, i.e. if $n=4$, then it is referred to as NP4.

\subsection{Surfactant Curvature}

Molecular geometry of surfactants plays an important role in the micellization process; therefore, it is essential to understand how surfactants pack. As described previously, two opposing forces control the self-association process: hydrocarbon/water 
interactions that favor aggregation (i.e., pulling surfactant molecules out of the aqueous environment), and head group interactions that work in the opposite sense. These two contributions can be considered an attractive interfacial tension term due to hydrocarbon tails and a repulsion term depending on the nature of the hydrophilic group. More recently, this basic idea was reviewed and quantified by Mitchell and Ninham ${ }^{60}$ and Israelachvili, ${ }^{61}$ resulting in the concept that aggregation of surfactants is controlled by a balanced molecular geometry. In brief, the geometric treatment separates the overall free energy of micellization into three critical geometric terms:

(1) $\mathrm{a}_{0}$ - the area occupied by the head group

(2) v-the volume of the hydrophobic tail

(3) $\quad 1_{\mathfrak{c}}$-the chain length of the tail

More generally, these variables define a critical packing parameter, $\mathrm{P}_{\mathrm{c}}$, as the ratio of the volume to surface area:

$$
P_{c}=\frac{v}{a_{0} l_{c}}
$$

The parameter $\mathrm{v}$ varies with the number of hydrophobic groups and chain branching, while $\mathrm{a}_{0}$ is mainly governed by head group hydration. 


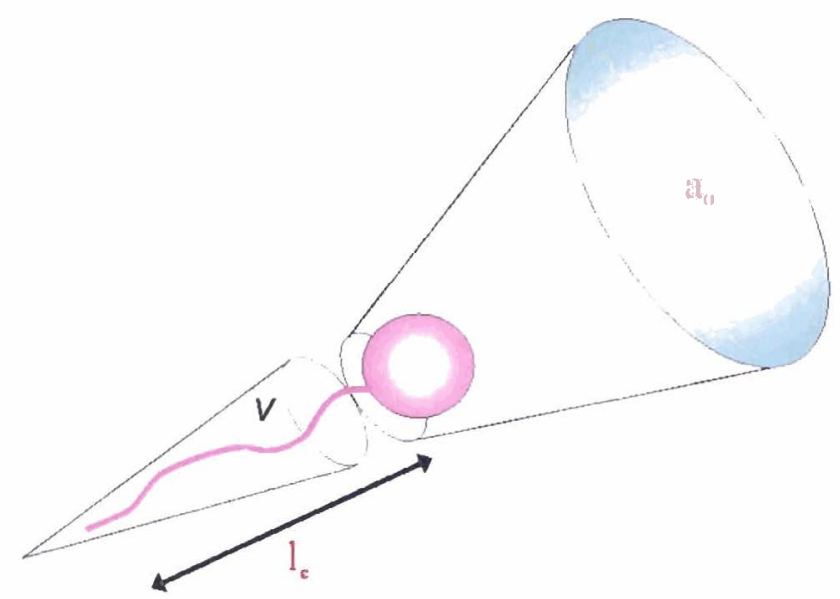

Figure 2.8: The critical packing parameter $P_{c}$ relates the head group area, the extended length and the volume of the hydrophobic part of a surfactant molecule into a dimensionless number $P_{c}=v / a_{0} l_{c}$.

The dimensionless value of $P_{c}$ is a useful quantity since it allows the prediction of aggregate shape and size. For example, surfactants with a $\mathrm{P}_{\mathrm{c}}$ greater than 1 are usually single-chained with large head groups (such as NP- $\mathbf{P}_{c}$ range $\left.0.96-3.0\right)^{62}$ and typically form reverse micelles. The surfactant geometry and system conditions influence the packing parameter, which controls the stability and curvature of the micelle. The curvature of surfactant molecules when forming a reverse micelle is one parameter that determines the micelle size. This is illustrated in Figure 2.9. 


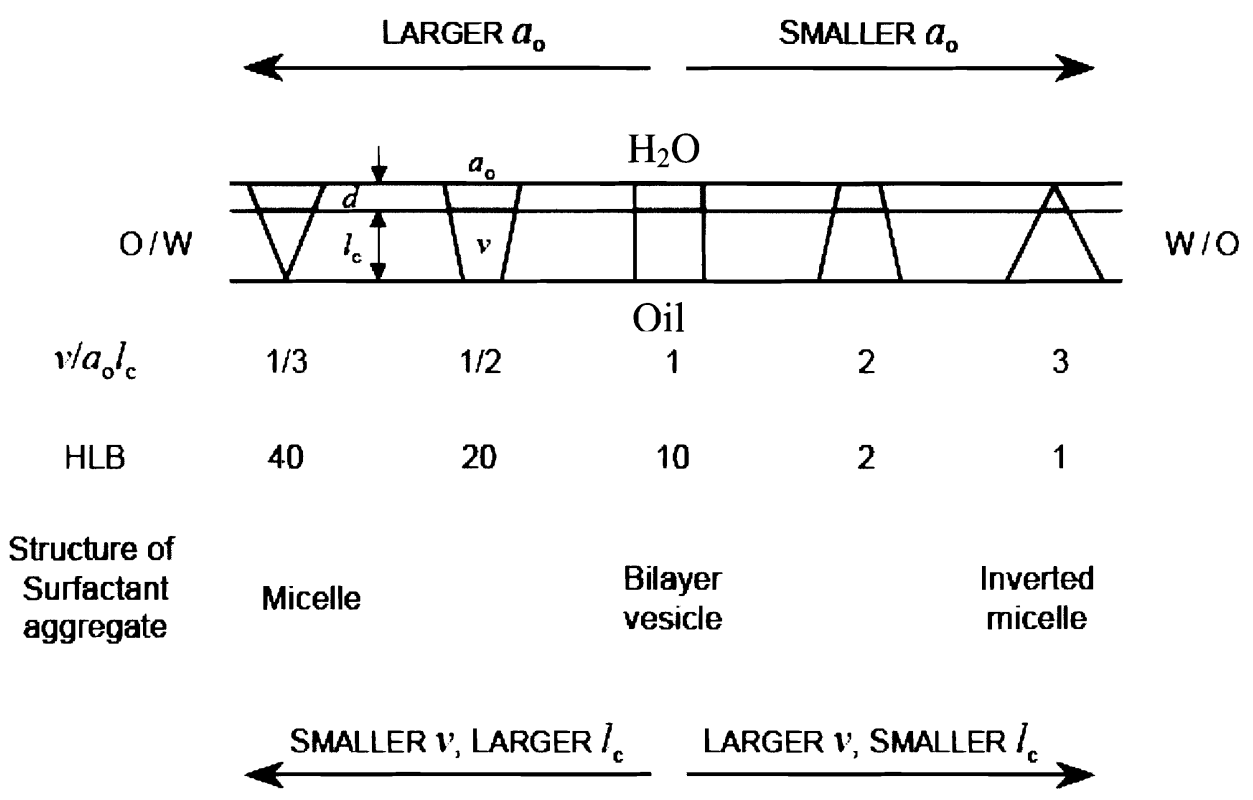

Figure 2.9: Effect of molecular geometry and system conditions on packing parameter.

Spontaneous curvature $\mathrm{C}_{\mathrm{o}}$ is defined as the curvature formed by a surfactant film when a system consists of equal amounts of water and oil. ${ }^{63}$ Then, there is no constraint on the film, which is free to adopt the lowest free energy state. Whenever one phase is predominant, there is a deviation from $\mathrm{C}_{\mathrm{o}}$. The curvature $\mathrm{C}_{\mathrm{o}}$ depends both on the composition of the phases it separates and on the surfactant type. One theory suggests that oil can penetrate to some extent between the surfactant hydrocarbon tails. The more extensive the penetration, the more curvature is imposed toward the polar side. This results in a decrease of $\mathrm{C}_{\mathrm{o}}$ since, by convention, positive curvature is toward oil (and negative is toward water). Recently, Eastoe, et al., have studied the extent of solvent penetration between surfactant molecules in microemulsions stabilized by nonionic surfactants using small angle neutron scattering (SANS) and selective deuteration. ${ }^{63}$ 
Results indicate that oil penetration is a subtle effect which depends on the chemical structures of both the surfactant and oil. In particular, unequal surfactant chain lengths result in a more disordered surfactant/oil interface, thereby providing a region of enhanced oil mixing. ${ }^{63-65}$ Although the use of a surfactant with a co-surfactant of different chain length is a commonly practiced technique, ${ }^{66-68}$ previous research has not elucidated on how micelle characteristics such as curvature, size and stability are affected by the ratio of the two. Therefore, the effect of changing the surfactant/total surfactant ratio (r) was explored in this study as well as all possible surfactant/co-surfactant combinations between the four commonly used NP's $(4,5,7$, and 9).

\subsection{Role of Co-surfactants}

The performance of a surfactant can be discussed in terms of effectiveness of adsorption to an interface. This is usually defined as the maximum lowering of surface tension $\gamma_{\min }$ regardless of concentration. In determining surfactant efficiency, the role of the molecular structure is primarily thermodynamic, while its role in effectiveness is directly related to the relative size of the hydrophilic and hydrophobic portions of the adsorbing molecule. The area occupied by each molecule is determined either by the hydrophobic chain cross-sectional area, or the area required for closest packing of head groups, whichever is greater. Therefore, surfactants can be tightly or loosely packed resulting in very different interfacial properties. For instance, straight chains and large head groups (relative to the tail cross section) favor close, effective packing, while 
branched, bulky, or multiple hydrophobic chains give rise to steric hindrance at the interface. ${ }^{65}$ For these reasons, it is interesting to investigate the use of a co-surfactant. Our preliminary dynamic light scattering (DLS) results indicated two combinations of surfactants clearly form reverse micelles-NP4/NP7 (HLB $\sim 7.5)^{69}$ and NP5/9 $(\mathrm{HLB} \sim 10)^{69}$. As a result, our investigation used IGEPAL® nonylphenol polyethoxylates 4 and 7 (NP4 and NP7)—-the same surfactant with different chain lengths. It has been shown that using NP7 with NP4 as a co-surfactant improves solubilization and overall micelle stability when compared to the corresponding single surfactants used for reverse micelle synthesis. ${ }^{33,36}$ In this case it is because NP4 provides a more uniform surface by filling in the gaps between the NP7 molecules, allowing surfactant molecules to pack tighter. ${ }^{70,71}$ Also, the stability of the water pools is primarily controlled by the hydrogen bonding association of the surfactant's head groups among themselves and/or with the aqueous cores of the droplets. Therefore, higher stability can be attained with more groups attaching to the micelle surfaces.

\subsection{Conclusions}

For the reasons discussed in this chapter, an NP4/NP7 reverse micelle system was the foundation of the central composite experimental design (CCD) as well as the basis of the investigation of the additional parameters. The effect of the hydrogen bonding system on the formation and size of reverse micelles was explored by substituting methanol and formamide for water. In addition, temperature and the presence and concentration of salts are suspected of disrupting the hydrogen bonding network, and 
were therefore also studied. The consequence of having a branched or cyclic hydrocarbon phase was considered by changing cyclohexane (commonly used for NP systems) to hexane, benzene, and isooctane. Widely accepted parameters proven to influence many systems of anionic and cationic reverse micelles are the surfactant concentration and $\omega$, the molar ratio of water to surfactant; hence, it is a good presumption that they will have similar effects on NP systems and so were also examined. Some experiments were repeated using a NP5/NP9 system so that the effects of surfactant chain length on micelle size could also be evaluated. Experiment descriptions and the details of the CCD will be discussed in Chapter 4, followed by discussion of the results of the CCD, models determined for both NP4/NP7 and NP5/NP9 systems, as well as the effects the aforementioned parameters have on reverse micelle size in Chapter 5. Before presentation of them, however, it is important to clarify and detail the characterization method used to determine reverse micelle size for the CCD in this work. 


\section{Chapter 3: Characterization}

A physicist, biologist and a chemist were going to the ocean for the first time.

The physicist saw the ocean and was fascinated by the waves. He said he wanted to do some research on the fluid dynamics of the waves and walked into the ocean. Obviously he was drowned and never returned.

The biologist said he wanted to do research on the flora and fauna inside the ocean and walked inside the ocean. He too, never returned.

The chemist waited for a long time and afterwards, wrote the observation, "The physicist and the biologist are soluble in ocean water." 


\subsection{Introduction}

Due to their small size and the fact that they are suspended in the liquid state, micelles present difficulties with respect to their characterization when compared to similar bulk or solid, dry materials. These difficulties are compounded when it is necessary to measure large quantities of various samples of different micelle sizes, which in turn can skew the results of complementary measurement techniques due to different weighting schemes used in averaging. In the development of a model that required over 50 experiments to predict reverse micelle size from synthetic conditions, it is important that the chosen particle sizing technique provide reverse micelle size and size distribution quickly and easily. This chapter briefly describes methods that can be used to determine these values followed by a detailed description of the technique employed in this workdynamic light scattering (DLS).

\subsection{Small Angle Scattering}

Small angle scattering techniques, including small angle X-ray scattering (SAXS) and small angle neutron scattering (SANS), are capable of giving information on the structural features of particles of colloidal size as well as their spatial correlation. Hence, they are very suitable for a comprehensive analysis of reverse micelles.

The physical principles of SAXS and SANS are the same. ${ }^{72}$ The electric field of the incoming wave induces dipole oscillations in the atoms. The accelerated charges generate secondary waves that add to the overall scattering amplitude. All secondary waves have the same frequency but may have different phases caused by different path 
lengths. A schematic description of this scattering experiment is shown in Figure 3.1. Xray (SAXS) or neutrons (SANS) from the source $T$ are formed into a fine beam, often by slits, and strike the sample $S$. A small fraction of this beam is scattered in other directions, e.g., in Figure 3.1 an angle $\theta$ with the direction of the incoming beam. $D$ is a detector, used to record the scattering intensity and its dependence on the scattering angle. Information about the structure of the sample can often be obtained from the analysis of the scattering intensity at a sequence of scattering angles.

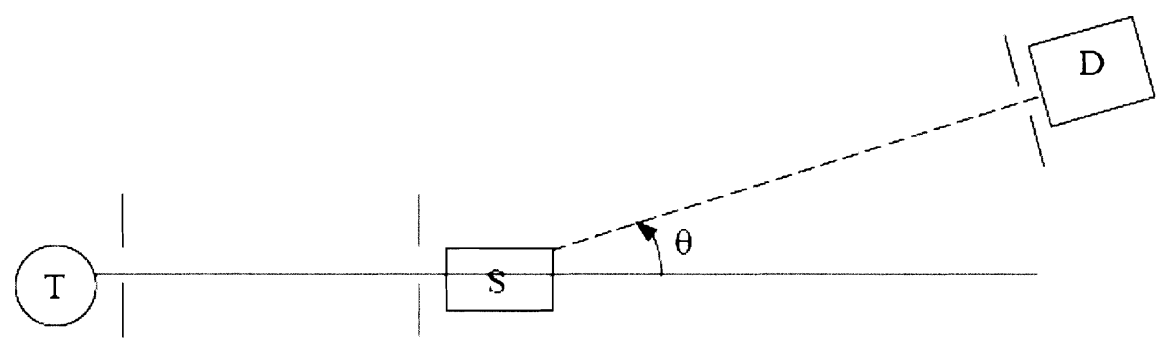

Figure 3.1: The essential parts of a small angle scattering system. The drawing shows the X-ray source $T$, the sample $S$, the scattering angle $\theta$, the slits used to define the incident and scattered beams, and the detector $D$.

Unlike other techniques, SAXS patterns do not give morphological information directly. The result of a SAXS experiment is essentially the intensity of the Fourier transform of the electron density and must be interpreted in order to determine morphology. One fundamental problem is that two different morphologies can, in theory, give identical scattering patterns. Generally, the exact microstructure cannot be reconstructed uniquely from a SAXS pattern because in a scattering experiment only the scattered radiation intensity can be measured and all phase information is lost. Therefore, a scattering pattern is not necessarily due to a particular morphology. 
SANS is a routine technique available at neutron-scattering facilities associated with research nuclear reactors. SANS differs from most other techniques in that it can only be carried out at large national or international facilities, there being no small scale 'home laboratory' version. Neutron sources are very expensive to build and to maintain. It costs millions of dollars annually to operate a nuclear research reactor and it costs that much in electrical bills alone to run a pulsed neutron source. Another problem with this technique is that neutron flux is very low. Presently, the neutron flux available at these reactors is equivalent to the $\mathrm{X}$-ray flux available in the 1940s. This makes for long measurement times and increased demand from researchers to use the facilities. In addition, the interaction of neutrons with matter is weak. Therefore, large samples are required. Although small angle scattering techniques provide beneficial data, these limitations exclude their use in this work.

\subsection{Dynamic Light Scattering}

Dynamic light scattering (DLS) theory is a well established technique for measuring micelle size over the range of a few nanometers to a few microns. The main advantage of DLS over rival particle sizing techniques is that size information can be obtained on the order of minutes. Furthermore, it is almost completely automated so that routine measurements are easily reproduced. Moreover, this method has modest development costs, takes less space and requires less money to purchase and maintain than other techniques that provide similar information. For these reasons, DLS is an ideal tool for measuring reverse micelle size for this work. 


\subsubsection{DLS Fundamentals}

If particles or droplets are illuminated by a laser having a known frequency, they will scatter the light in all directions but at different frequencies. The change in the frequency is quite similar to the change in frequency or pitch one hears when an ambulance with its wailing siren approaches and finally passes. This shift is termed a Doppler shift, and the concept is the same for light when it interacts with small moving particles. For the purposes of micelle size measurement, the shift in light frequency is related to the size of the micelles causing the shift.

If a screen is held close to particles or droplets illuminated by a laser, the screen will be illuminated by the scattered light and will show a speckle pattern (see Figure 3.2) consisting of areas of bright light and dark areas where no light is detected.

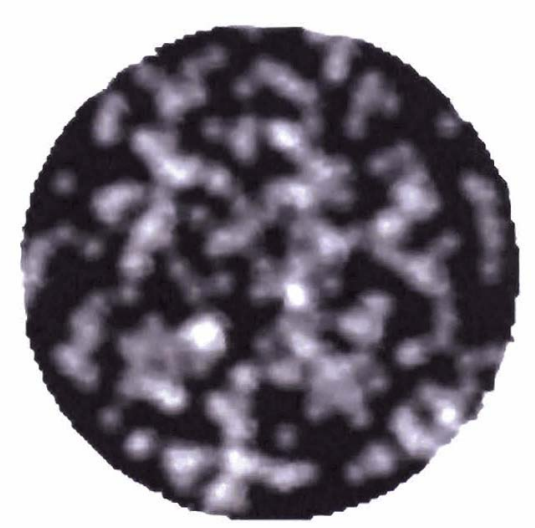

Figure 3.2: Speckle pattern consisting of bright light and dark areas from constructive and destructive interference, respectively. ${ }^{73}$

The bright areas of light are where the light scattered by the micelles arrive at the screen with the same phase and interferes constructively to form a bright patch. The dark areas are where the phase additions are mutually destructive and cancel each other out. 


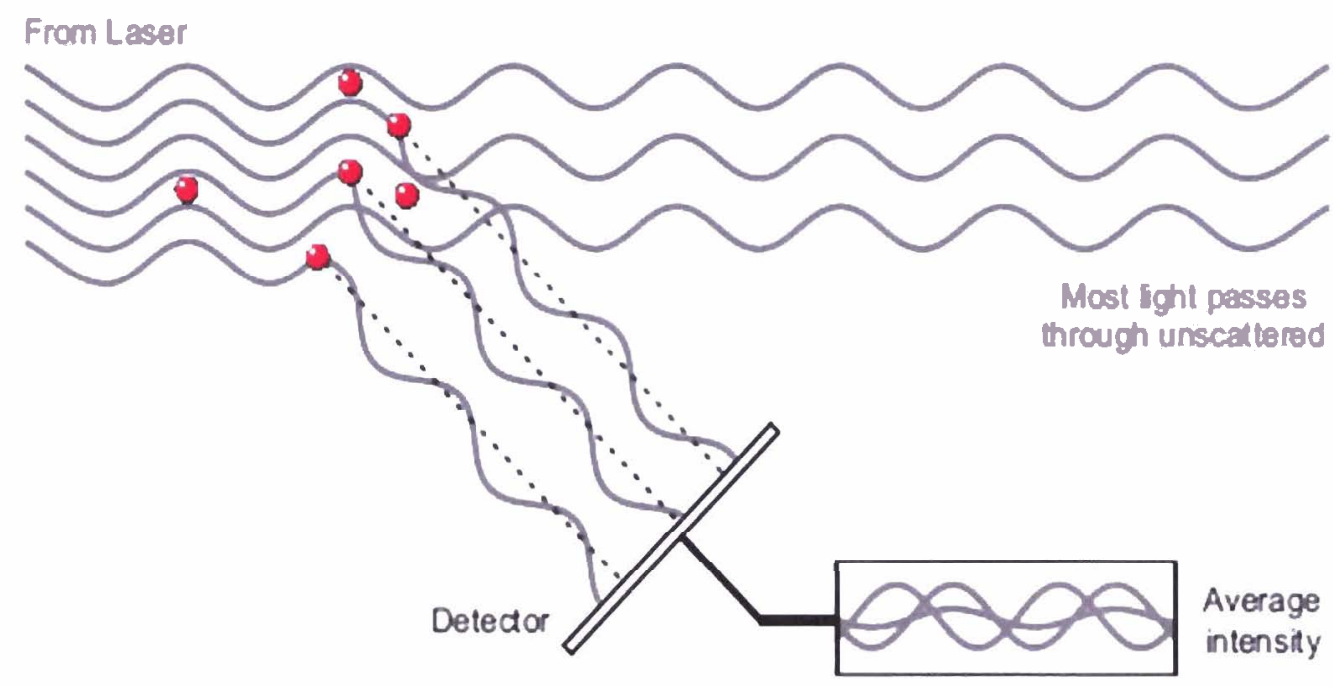

Figure 3.3: Propagated waves from the light scattered by particles. Bright areas of light are from particles arriving at the screen with the same phase and they interfere constructively to form a bright patch. The dark areas are where the phase additions are mutually destructive and cancel each other out. ${ }^{73}$

In practice, micelles suspended in a liquid are constantly moving due to Brownian motion - the movement of particles due to random collisions with the molecules of the surrounding liquid. The velocity of this Brownian motion is the translational diffusion coefficient. An important feature of Brownian motion is that small micelles move quickly and large micelles move more slowly. If large micelles are being measured, then the intensity of the speckle pattern (see Figure 3.2) will fluctuate slowly since they are moving slowly. Similarly, if small micelles are being measured, then the intensity of the speckle pattern will fluctuate quickly. As the micelles are constantly in motion, the speckle pattern produced from the illuminated micelles will also appear to move. As the micelles move around, the constructive and destructive phase addition of the scattered light will cause the bright and dark areas to fluctuate in intensity. 
The angular dependence of the sample scattering intensity arises from constructive and destructive interference of light scattered from different positions on the same particle. This phenomenon is known as Mie scattering and occurs when the particle is large enough to accommodate multiple photon scattering. ${ }^{74}$ When the particles in solution are much smaller than the wavelength of the incident light (633 nm in this case), multiple photon scattering will be avoided. Under these conditions, the angular dependence of the scattering intensity is lost. This type of scattering is known as Rayleigh scattering. ${ }^{75}$ Therefore, when particles are very small compared with the wavelength of the light, the intensity of the scattered light is uniform in all directions (Rayleigh scattering); for larger particles (above approximately $250 \mathrm{~nm}$ diameter), the intensity is angle-dependent (Mie scattering). Since the reverse micelles measured for this study are smaller than the wavelength of the laser, the intensity of the scattered light is independent of the angle and is thus Rayleigh scattering.

\subsubsection{Correlator Function}

Within the DLS instrument is a digital correlator that measures the degree of similarity between two signals over a period of time. If the intensity signal of a particular part of the speckle pattern at one point in time was compared to the intensity signal a very short time later, the two signals would be very similar-or strongly correlated. If the original signal was then compared to a signal a little further ahead in time, there would still be a relatively good comparison between the two signals, but it would not be as good as the first. The correlation is therefore decreasing with time. Now consider the initial 
intensity of the signal with the intensity at a much later time - the two signals would have no relation to each other since the particles are moving in random directions (due to Brownian motion). In this situation it is said that there is no correlation between the two signals.

Recall from Chapter 2 that the fast exchange of monomers between micelles and the surrounding bulk phase is on the order of microseconds ${ }^{55}$ and the micelle formation and dissociation process is usually on the order of milliseconds. ${ }^{55}$ DLS deals with very small time scales. In a typical speckle pattern, the length of time it takes for the correlation to reduce to zero is on the order of 1 to 10 's of milliseconds. The "short time later" is on the order of nanoseconds or microseconds. ${ }^{73}$ This timeframe provides an excellent means of measuring the size of reverse micelles since the instrument is faster than both relaxation processes. If the initial signal intensity were compared with itself then there would be perfect correlation because the signals are identical. Perfect correlation is reported as 1 and no correlation is reported as 0 . Figure 3.4 shows the correlation function for large and small particles. As can be seen, the rate of decay for the correlation function is related to particle size since the rate of decay is much faster for small particles than it is for larger particles. 


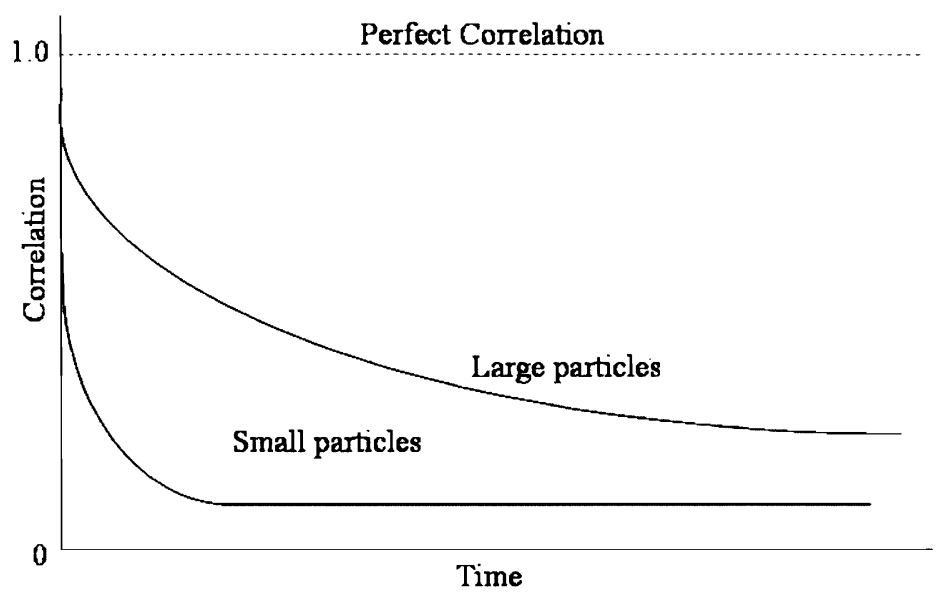

Figure 3.4: A typical correlation function of correlation against time showing the rate of decay for the correlation function is related to particle size - the rate of decay is much faster for small particles than it is for large. ${ }^{73}$

Each monodisperse population of particle sizes produces its own unique autocorrelation function - a single exponential decay. Mixtures of more than one size population produce sums of exponentials. The autocorrelation function, $\mathrm{C}(\tau)$, is shown in equation 3.1 where $\mathrm{A}$ and $\mathrm{B}$ are instrumental constants - $\mathrm{A}$ is the amplitude or intercept of the correlation function and B is the baseline.

$$
C(\tau)=A e^{-2 \Gamma_{t}}+B
$$

The correlation function can then be used to calculate the size distribution of the sample.

In dynamic light scattering, Z-average size (also known as the "cumulants mean") is the most important and stable number produced by the technique. This is the size that should be used if a number is required for quality control purposes. It will only be comparable with other techniques if the sample is monomodal (i.e., only one peak), spherical and monodisperse (i.e., no width to the distribution). In any other case, the Zaverage size can only be used to compare results with samples measured in the same 
dispersant, by the same technique, i.e., by DLS. The cumulants analysis only gives two values - a mean value for the size and a width parameter known as the polydispersity, or the polydispersity index (PDI). It is important to note that this mean size (often given the symbol Z or Z-average) is an intensity mean-not a mass or number mean-because it is calculated from the signal intensity. The cumulants analysis is actually the fit of a polynomial to the log of the correlation function.

$$
\ln C(\tau)=a+b t+c t^{2}+\mathrm{dt}^{3}+\mathrm{et}^{4}+\ldots \ldots \ldots \ldots
$$

The value of $\mathrm{b}$ is known as the second order cumulant, or the Z-average diffusion coefficient. This is converted to a size using the dispersant viscosity and some instrumental constants. Only the first three terms - a, b, and c-are used in the standard analysis to avoid over-resolving the data; however this does mean that the Z-average size is likely to be interpreted incorrectly if the distribution is very broad (i.e. has a high polydispersity).

In the cumulants analysis, a single particle size distribution is assumed and a single exponential fit is applied to the autocorrelation function. It is important to note that the cumulants analysis algorithm does not yield a distribution — it gives only the intensity weighted "Z-average" mean size and the PDI. If one were to assume a single size population following a Gaussian distribution, then the PDI would be related to the standard deviation of the hypothetical Gaussian distribution according to the following equation.

$$
P D I=\frac{\sigma^{2}}{Z^{2}}
$$


From the decay rate of the correlation function $\Gamma$, the diffusion coefficient can be derived using the following equation where $\mathrm{q}$ is the scattering vector and $\mathrm{D}$ is the diffusion coefficient.

$$
\Gamma=q^{2} D=\left(\frac{2 \pi n}{\lambda} \sin \frac{\theta}{2}\right)^{2} \times \frac{k_{B} T}{6 \pi \eta d}
$$

From the measured diffusion coefficient $\mathrm{D}$, the hydrodynamic diameter $\mathrm{d}$ can be calculated from equation 3.4 where $n$ is the refractive index, $\lambda$ is the laser wavelength, $\theta$ is the scattering angle, $\mathrm{k}_{\mathrm{B}}$ is Boltzmann's constant, $\mathrm{T}$ is the absolute temperature, and $\eta$ is the liquid viscosity. Figure 3.5 shows a plot of the natural log of the autocorrelation function versus the scattering vector. The result is a straight line with a slope of $-2 \mathrm{D}$.

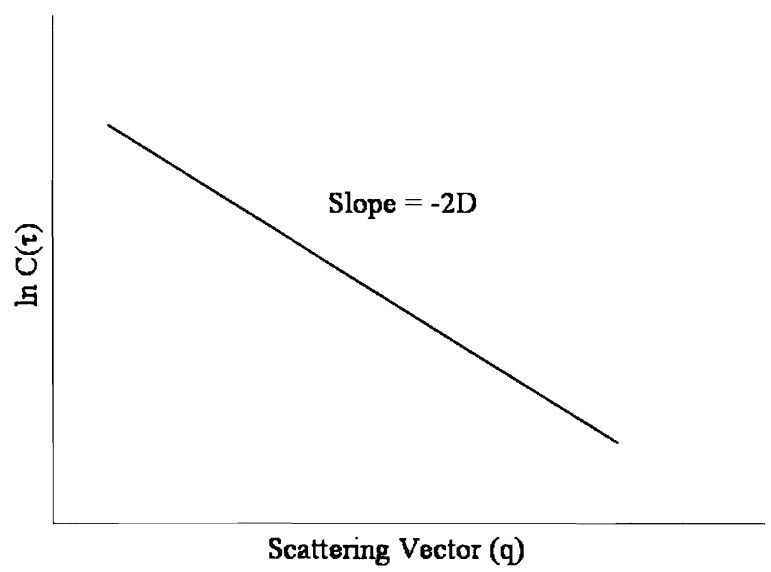

Figure 3.5: Relationship of correlation function $\mathrm{C}(\tau)$ and translational diffusion coefficient (D).

The relationship between the hydrodynamic diameter, $d$, of a particle or droplet and its speed due to Brownian motion can be simplified to the Stokes-Einstein equation shown below. 


$$
d=\frac{k_{B} T}{3 \pi \eta D}
$$

The DLS software uses algorithms to extract the decay rates for a number of size classes to produce a size distribution. A typical size distribution graph known as an intensity distribution is shown below where the $\mathrm{X}$ axis shows a distribution of size classes, while the $\mathrm{Y}$ axis shows the relative intensity of the scattered light.

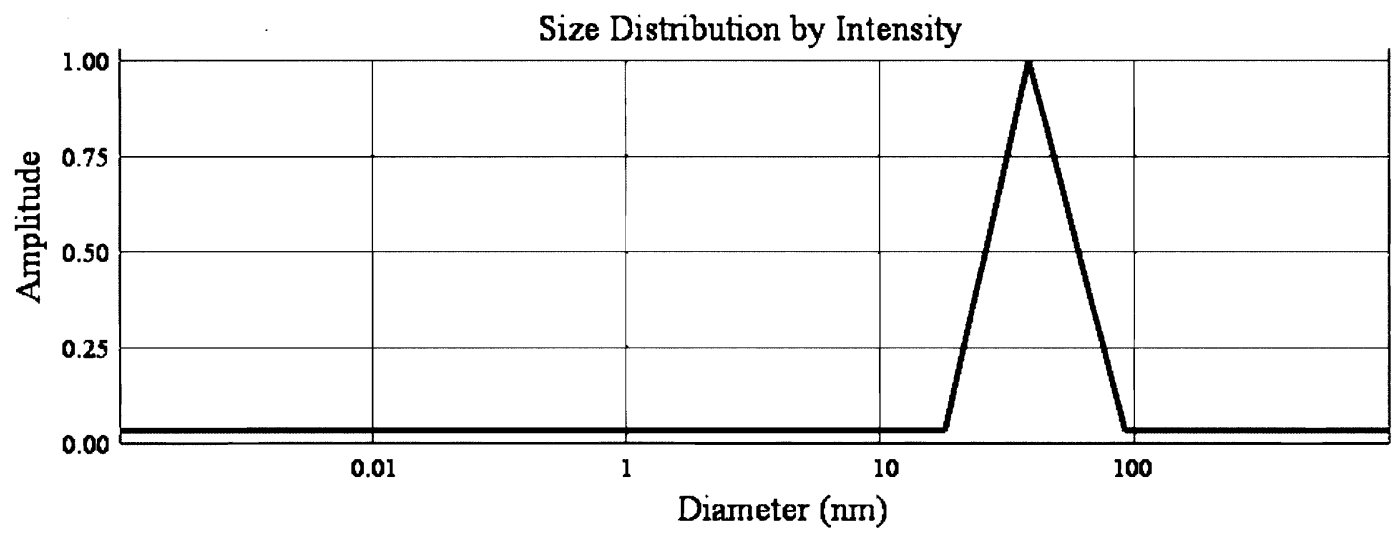

Figure 3.6: A typical size distribution graph where the $\mathrm{X}$ axis shows size classes, while the $\mathrm{Y}$ axis shows the relative intensity of the scattered light. This is therefore known as an intensity distribution. ${ }^{73}$

The basic distribution obtained from a DLS measurement is intensity-all other distributions are generated from this using Mie theory. ${ }^{73}$ The intensity distribution can be converted to a volume distribution, which can be further converted to a number distribution. However, number distributions are of limited use because small errors in gathering data for the correlation function leads to huge errors in distribution by number. A truly monodisperse sample would give rise to a single exponential decay to which fitting a calculated particle size distribution is relatively straightforward. In practice, polydisperse samples give rise to a series of exponentials requiring several quite complex 
schemes to be devised for the fitting process. One of the methods most widely used for polydisperse samples is known as Non-Negatively Constrained Least Squares (NNLS) and is used by Malvern Instruments Zetasizer Nano ZS digital correlator software. ${ }^{76-78}$ The particle size distribution from DLS is derived from a deconvolution of the measured intensity autocorrelation function of the sample as discussed earlier (equation 3.1). Generally, this deconvolution is accomplished using a NNLS fitting algorithm; common examples are CONTIN, Regularization, and the General Purpose and Multiple Narrow Mode algorithms included in the Zetasizer Nano software.

\subsubsection{Instrumentation}

A typical DLS system is comprised of six main components and is shown in Figure 3.7. A laser (1) is used to provide a light source to illuminate the sample particles within a cell (2). Most of the laser beam passes straight through the sample, but some is scattered by the particles within the sample. A photodiode detector (3) is used to measure the intensity of the scattered light. As a particle scatters light in all directions, it is (in theory), possible to place the detector in any position to detect the scattering. With the Zetasizer Nano Series, depending upon the particular model, the detector position will be at either $173^{\circ}$ or $90^{\circ}$. For the Zetasizer Nano Series ZS used in this study, the detector position was at $173^{\circ}$. 

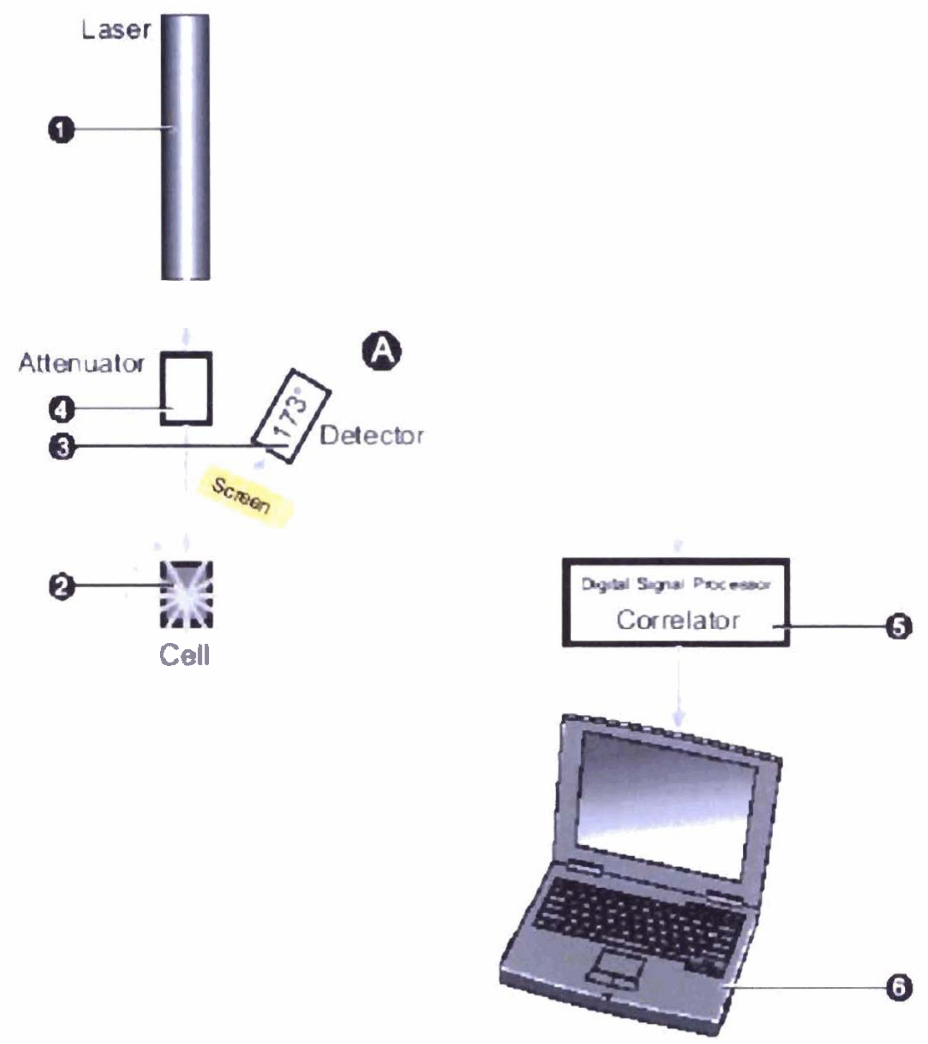

Figure 3.7: Schematic of a typical DLS system showing its six main components: (1) laser; (2) sample cell; (3) detector; (4) attenuator; (5) correlator; and (6) computer. ${ }^{73}$

The intensity of the scattered light must be within a specific range for the detector to successfully measure it. If too much light is detected then the detector will become overloaded. To overcome this, an "attenuator" (4) is used to reduce the intensity of the laser and hence reduce the intensity of the detected light.

For samples that do not scatter much light, such as very small particles or samples of low concentration, the amount of light striking the detector should be maximized. In this situation, the attenuator will allow more laser light through to the sample. For samples that scatter more light, such as large particles or samples of higher concentration, the amount of light striking the detector must be decreased. This is achieved by using the 
attenuator to reduce the amount of laser light that passes through to the sample. The appropriate attenuator position is automatically determined by the instrument during the measurement sequence.

The scattering intensity signal for the detector is passed to a digital signal processing board called a correlator (5). The correlator compares the scattering intensity at successive time intervals to derive the rate at which the intensity is varying. This correlator information is then passed to a computer (6), where the software will analyze the data and derive size information.

As mentioned earlier, the detection optics of the Zetasizer Nano model used in this study was in the $173^{\circ}$ position. This is known as backscatter detection. There are several advantages to doing this. Because the backscatter is being measured, the incident beam does not have to travel through the entire sample. This reduces an effect known as multiple scattering, where the scattered light from one particle is itself scattered by other particles. As the light passes through a shorter path length of the sample, then higher concentrations of sample can be measured.

Contaminants such as dust particles within the dispersant are typically large compared to the sample size. Large particles mainly scatter in the forward direction. Therefore, by measuring the backscatter, the effect of dust is greatly reduced. The effect of multiple scattering is at a minimum at about $180^{\circ}$ - this allows higher concentrations to be measured. 


\subsubsection{DLS Performance}

In the present work, the size of the reverse micelles was characterized in terms of the hydrodynamic diameter. DLS measurements were made at $25^{\circ} \mathrm{C}$ (with the exception of temperature dependent data) with a Malvern Instruments Zetasizer Nano ZS to estimate the hydrodynamic diameter of the reverse micelles. The refractive index and viscosity used for cyclohexane were 1.4262 and $0.894 \mathrm{mPa} \cdot \mathrm{s}$ respectively, while the refractive index used for water and formamide were 1.333 and 1.447 , respectively. ${ }^{79}$

A potential problem with DLS is that the translational diffusion coefficient is affected by other parameters such as ionic strength and shape, resulting in inaccurate measurements. In order to verify the performance of the Zetasizer Nano ZS, a comparison to reference data from a previous study was carried out. Herrera investigated AOT reverse micelles as a templating system for the production of gold nanoparticles. ${ }^{80}$ In his experiments, DLS experiments were compared to TEM and time-correlated UVvis absorption measurements, two commonly accepted methods of size determination. For instrument verification, AOT reverse micelles were prepared similarly to Herrera and sizes were determined via DLS and compared to those obtained by Herrera. ${ }^{80}$

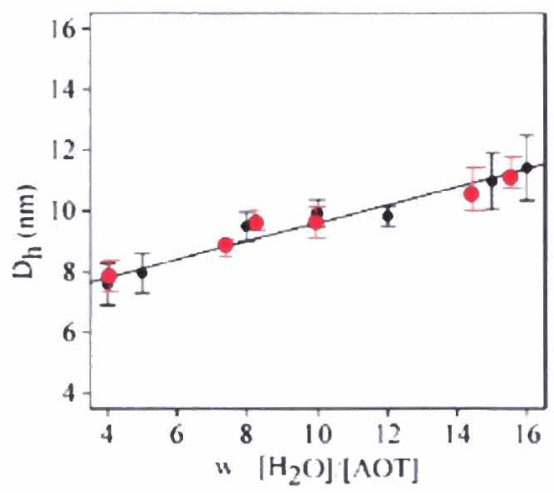

Figure 3.8: AOT/isooctane reverse micelle data obtained via DLS overlaid over Herrera's AOT/isooctane reverse micelles to verify instrument performance. 
As shown in Figure 3.8, accepted AOT micelle sizes fell within the standard deviation size range of the obtained AOT reverse micelles, validating this method for determining reverse micelle sizes. 
Chapter 4: Central Composite Design and Statistical Analysis

A chemical is a substance that:

- An organic chemist turns into a foul odor.

* An analytical chemist turns into a procedure.

- A physical chemist turns into a straight line.

* A biochemist turns into a helix.

* A chemical engineer turns into a profit. 
4.1 Introduction

Since DLS allows the determination of reverse micelle size quickly and easily, a response surface to correlate size with synthetic conditions is easily obtained. Response surface methods are used to examine the relationship between one or more response variables and a set of quantitative experimental variables or factors. ${ }^{32}$ These methods are often employed after a "vital few" controllable factors have been identified and the factor settings that optimize the response are desired. Response surface methods may be used to find factor settings that produce the "best" response or satisfy operating or process specifications, to identify new operating conditions that produce demonstrated improvement in, or to model a relationship between the quantitative factors and the response. Central composite designs (CCDs) are response surface designs that can fit a full quadratic to model this relationship. ${ }^{32}$ Therefore, to model the relationship between synthetic conditions and reverse micelle size including any second-order and interaction effects, a CCD was employed and will be discussed in greater detail.

\subsection{Central Composite Design}

CCDs offer a more systematic approach compared to other ways of examining all factors at several levels over the full range of the chosen "reaction space." Importantly, the data collected from a CCD also allows the generation of a mathematical model (response surface model) of the chemical process based on the statistical analysis of this set of designed experiments. ${ }^{7}$ This model then allows for the analysis of interactions 
between the factors and hence offers the opportunity to achieve better understanding and control of the experimental system.

There are CCDs for any number of factors, but the focus here will be on the three factor case. A collection of data for three variables can be graphically represented in three dimensions by a cube. The CCD is called composite because it can be thought of as the union of three separate designs: (1) the eight corners of the cube, which form a two level full factorial; (2) the six points in the centers of each face, known as the axial points or the star points; and (3) the center point. It is called central to distinguish it from noncentral designs in which the centroid of the star is displaced relative to the center of the cube. $^{81}$

In a multivariate experiment, all of the variables are changed during each run. The need for this arises because the variables often interact with each other. For example, if ionic strength conditions are painstakingly optimized at one surfactant concentration, this work may have to be repeated if it is subsequently found that a different concentration works better. In a multivariate approach, variation of surfactant concentration would have been included in the first round of experiments. This would have shown the best direction to move within the multidimensional space defined by the variables.

To visualize a CCD, consider several experimental variables that can vary between low and high values. For convenience, the factors are scaled from -1 to +1 . One CCD consists of cube points at the corners of a unit cube that is the product of the intervals $[-1,1]$, star points along the axes at or outside the cube, and center points at the 
origin. ${ }^{82}$ This juxtapositioned circumscribed cube and star design allows the estimation of the interaction parameters as well as second-order parameters, respectively.

A five-level, three-factor CCD was employed in this study, requiring 23 experiments, as shown in Figure 4.1. The design consisted of 8 factorial points (cube), 12 axial points ( 2 axial points on the axis of each design variable as well as 2 additional points along each axis forming a star), and 3 center points. As seen in Figure 4.1, each corner of the box represents a set of factors. Changing factors from one corner to another along the axis gives the first order response of the micelle size. Moving diagonally provides the information on the effect from the interactions among factors. ${ }^{7}$ The star is added to provide the axial points for testing the possible second-order effects of the factors. It is important to note that an asymmetric design was used due to the feasible range of the parameters and to concentrate on the conditions within the experimental reaction space in which reverse micelles are formed. 


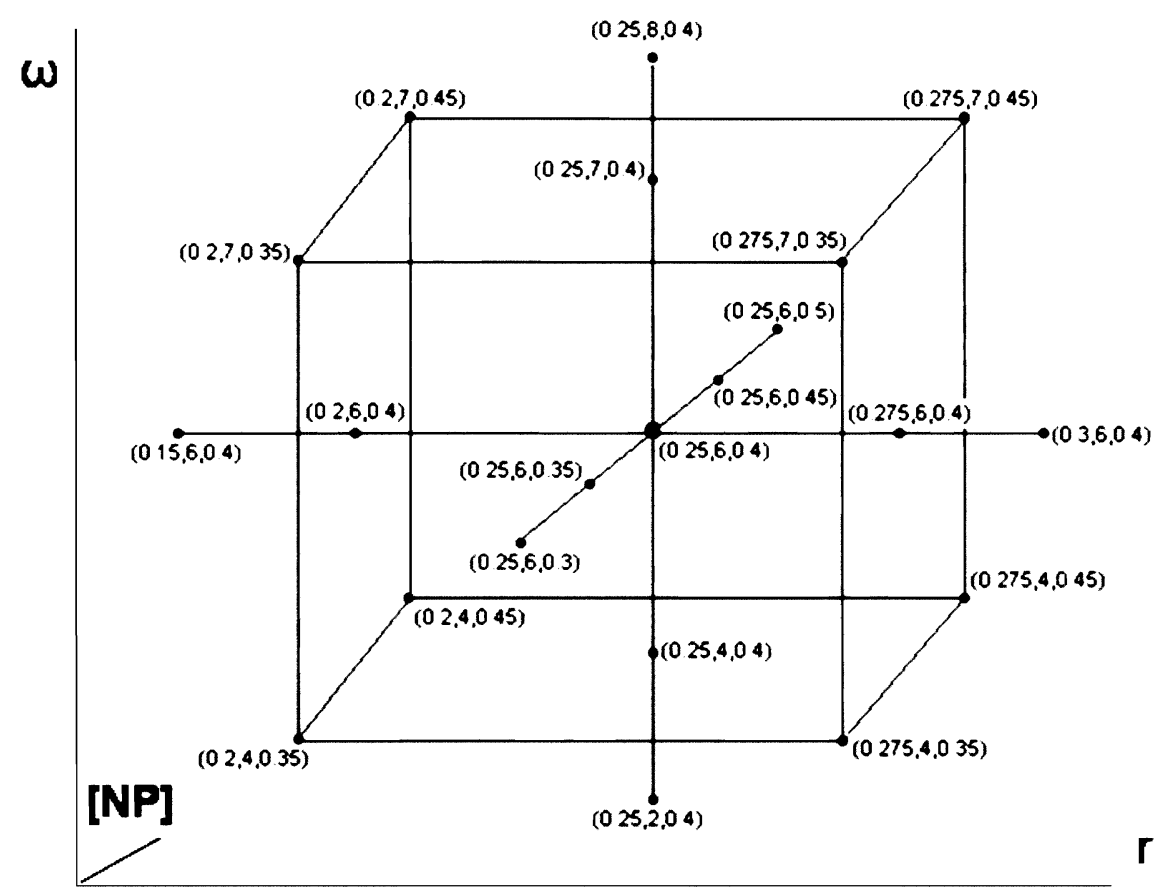

Figure 4.1: Asymmetric central composite design of superimposed box and star designs ( $\mathrm{r}, \omega,[\mathrm{NP}])$ illustrating experiments for determination of interaction and second-order parameters.

\subsection{Phase Space}

Initial single variable experiments were carried out to identify the most important control factors and the experimentally feasible ranges for the experiments. From these experiments, $r, \omega$, and $[\mathrm{NP}]$ were selected as the variables to include in the CCD. Microemulsion solubilization experiments were performed by titrating the necessary amount of water into a mixture of NP4 and NP7 and cyclohexane (total volume approximately $10 \mathrm{~mL}$ ) to obtain the desired value of $\omega$. Recall the phase diagram presented earlier (see Figure 4.2). For the initial screening experiments, water was added until the solutions were no longer optically transparent to visually determine the region in which reverse micelles form and therefore to select the levels for the CCD. The onset of 
solution turbidity and the subsequent increase in viscosity indicated that the maximum water solubilization in the reverse micelle region had been exceeded.

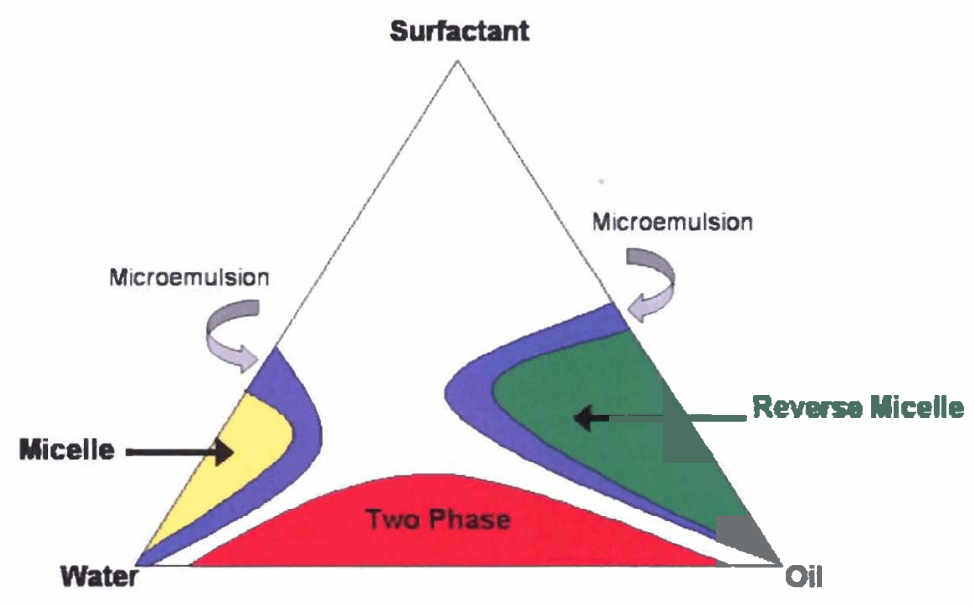

Figure 4.2: Pseudo phase diagram of surfactant, water and oil illustrating the regions in which micelles and reverse micelles form.

In addition, the fact that the micelle sizes are several times bigger than the surfactant molecular length $(2-3 \mathrm{~nm})$ reflects micellization of surfactant molecules in solution. Similar screening experiments to determine the corresponding phase space for NP5 and NP9 were performed.

The parameters for the box and star design are shown in Table 4.1. Initially, the surfactant concentration range examined was $0.1 \mathrm{M}$ to $0.5 \mathrm{M}$ and centered at $0.3 \mathrm{M}$. Concentrations below $0.3 \mathrm{M}$, however, showed very large sizes indicating the $\mathrm{CMC}$ had not been exceeded—surfactant existed as monomers. Therefore, the surfactant concentration axis was shifted and reexamined centered on $0.4 \mathrm{M}$. Data from the original star design can be seen in Table 4.2. In addition to the re-centered star points, these points were also included in the model determination for NP4/NP7 reverse micelles since they are within the constraints of the phase space. 
Table 4.1: Experimental conditions for box and star CCD containing 23 points -13 star, 8 box, as well as 2 additional center replicates. $\omega$ varied from $2-8$; [NP] varied from $0.3 \mathrm{M}-0.5 \mathrm{M}$; and $\mathrm{r}$ varied from 0.15 0.30. Predicted sizes were obtained from equation 4.2.

\begin{tabular}{|c|c|c|c|c|c|c|c|}
\hline Solution & $\mathbf{r}$ & $\boldsymbol{\omega}$ & $\boldsymbol{I N P ]}$ & Ave Size (nm) & Std Dev & Predicted Size (nm) & \% Error \\
\hline Star 1 & 0.15 & 6.0 & 0.40 & 11.32 & 0.96 & 9.40 & -17.0 \\
\hline Star 2 & 0.20 & 6.0 & 0.40 & 8.21 & 0.34 & 8.33 & 1.45 \\
\hline Star 3 (center) & 0.25 & 5.9 & 0.40 & 5.65 & 0.14 & 7.25 & 28.4 \\
\hline Star 4 & 0.27 & 6.0 & 0.40 & 7.49 & 0.15 & 7.05 & -5.92 \\
\hline Star 5 & 0.30 & 6.0 & 0.40 & 5.90 & 0.36 & 6.34 & 7.48 \\
\hline Star 6 & 0.25 & 6.0 & 0.30 & 8.74 & 0.46 & 10.2 & 16.8 \\
\hline Star 7 & 0.25 & 6.0 & 0.34 & 8.37 & 0.44 & 9.07 & 8.39 \\
\hline Star 8 & 0.25 & 6.0 & 0.45 & 5.15 & 0.08 & 7.27 & 41.1 \\
\hline Star 9 & 0.25 & 6.0 & 0.50 & 6.73 & 0.73 & 4.67 & -30.7 \\
\hline Star 10 & 0.25 & 8.0 & 0.40 & 12.98 & 2.16 & 9.26 & -28.6 \\
\hline Star 11 & 0.25 & 6.9 & 0.40 & 7.35 & 0.47 & 8.23 & 12.0 \\
\hline Star 12 & 0.25 & 4.0 & 0.40 & 6.46 & 0.31 & 5.50 & -14.8 \\
\hline Star 13 & 0.25 & 2.0 & 0.40 & 3.87 & 0.07 & 3.61 & -6.69 \\
\hline Center rep 2 & 0.25 & 6.0 & 0.40 & 5.63 & 0.13 & 7.25 & 28.8 \\
\hline Center rep 3 & 0.25 & 6.0 & 0.40 & 5.61 & 0.13 & 7.25 & 29.2 \\
\hline Box 1 & 0.20 & 4.0 & 0.35 & 6.08 & 0.79 & 5.50 & -14.8 \\
\hline Box 2 & 0.20 & 4.0 & 0.45 & 5.99 & 0.73 & 3.61 & -6.69 \\
\hline Box 3 & 0.21 & 7.0 & 0.35 & 9.69 & 0.72 & 7.07 & 16.2 \\
\hline Box 4 & 0.20 & 7.0 & 0.45 & 6.68 & 0.12 & 5.26 & -12.2 \\
\hline Box 5 & 0.27 & 4.0 & 0.35 & 5.81 & 0.62 & 11.0 & 13.2 \\
\hline Box 6 & 0.27 & 4.0 & 0.45 & 5.39 & 1.21 & 8.05 & 20.5 \\
\hline Box 7 & 0.27 & 7.0 & 0.35 & 8.11 & 0.34 & 6.17 & 6.28 \\
\hline Box 8 & 0.27 & 7.0 & 0.45 & 5.46 & 0.07 & 4.35 & -19.2 \\
\hline
\end{tabular}

Table 4.2: Original star design centered at $[\mathrm{NP}]=0.3 \mathrm{M}$. Additional star points used to calculate the model for NP4 and NP7 (equation 4.2).

\begin{tabular}{|c|c|c|c|c|c|c|c|}
\hline Solution & $\mathbf{r}$ & $\boldsymbol{\omega}$ & {$[\mathbf{N P}]$} & Ave Size (nm) & Std Dev & Predicted Size (nm) & \% Error \\
\hline Star 1 & 0.20 & 3.0 & 0.31 & 4.99 & 0.27 & 6.51 & 30.5 \\
\hline Star 2 & 0.25 & 3.0 & 0.30 & 5.25 & 0.19 & 5.90 & 12.5 \\
\hline Star 3 & 0.30 & 3.1 & 0.30 & 5.18 & 0.19 & 5.59 & 7.81 \\
\hline Star 4 & 0.36 & 3.1 & 0.30 & 4.89 & 0.02 & 5.04 & 3.01 \\
\hline Star 5 & 0.40 & 3.1 & 0.30 & 4.92 & 0.04 & 4.52 & -8.00 \\
\hline Star 6 & 0.25 & 3.0 & 0.30 & 5.25 & 0.19 & 5.90 & 12.5 \\
\hline Star 7 & 0.25 & 6.1 & 0.30 & 12.2 & 0.53 & 10.3 & -15.9 \\
\hline Star 8 & 0.25 & 4.2 & 0.30 & 9.56 & 0.68 & 7.58 & -20.7 \\
\hline Star 9 & 0.25 & 5.1 & 0.30 & 7.91 & 2.91 & 8.84 & 11.7 \\
\hline Star 10 & 0.26 & 2.1 & 0.31 & 5.90 & 0.05 & 4.54 & -23.0 \\
\hline Star 11 & 0.25 & 6.1 & 0.30 & 12.2 & 0.53 & 10.2 & -16.8 \\
\hline Star 12 & 0.25 & 5.9 & 0.25 & 13.3 & 0.09 & 11.3 & -14.5 \\
\hline Star 13 & 0.25 & 6.0 & 0.24 & 12.5 & 0.11 & 11.9 & -5.06 \\
\hline
\end{tabular}




\subsection{Statistical Analysis}

The factor level limits (i.e., maximum and minimum values) were determined by initial screening experiments as discussed earlier in which samples with minimal micelle size and maximum water solubilization were synthesized. Once these were established, the independent factors, levels, and experimental design were selected, as shown in Table 4.1. A 10-parameter model represented by the following second-order polynomial equation was fit to the data and included up to second-order effects in each factor and all two-factor interactions between the three factors. ${ }^{83}$

$$
Y=\beta_{0}+\sum_{i=1}^{3} \beta_{i} x_{i}+\sum_{i=1}^{3} \beta_{i j} x_{i} x_{j}+\sum_{i=1}^{3} \beta_{i i} x_{i}^{2}
$$

In this equation, $\mathrm{Y}$ is the response (reverse micelle size); $\beta_{0}, \beta_{\mathrm{i}}, \beta_{\mathrm{ij}}$, etc. are constant coefficients; and the $\mathrm{x}_{\mathrm{i}}$ are the uncoded independent variables (r, $\omega$, and [NP]).

ANOVA is used to uncover both the main and the interaction effects of independent variables on a dependent variable, reverse micelle size in this case. A "main effect" is the direct effect of an independent variable on the dependent variable. An "interaction effect" is the joint effect of two or more independent variables on the dependent variable. ${ }^{32}$

The key statistic in ANOVA is the F-test of difference of group means, which tests if the means of the groups formed by values of the independent variable (or combinations of values for multiple independent variables) are different enough not to have occurred by chance. If the group means do not differ significantly then it is inferred that the independent variable(s) did not have an effect on the dependent variable. As 
previously mentioned, main effects are the unique effects of the independent variables. If the probability associated with the calculated $\mathrm{F}$ statistic is less than 0.05 for any independent variable, it is concluded that that variable does have an effect on the dependent variable. Interaction effects are the joint effects of pairs, triplets, or higherorder combinations of the independent variables, different from what would be predicted from any of the independent variables acting alone. That is, when there is an interaction, the effect of an independent variable on a dependent variable varies according to the values of another independent variable. If the probability associated with the calculated $\mathrm{F}$ statistic is less than 0.05 for any such combination of variables, it is concluded that their interaction does have an effect on the dependent variable. The concept of interaction between two independent variables is related to their joint effect on the dependent variable, whereas correlation indicates whether or not they can be used as separate variables in the model.

The micelle size as a function of the independent variables $r, \omega$, and [NP] was modeled using stepwise regression in the statistical program SPSS Version 13. Stepwise functionality was used to find the best predictor from a number of possible predictors by adding and removing variables in steps until the optimum model was reached in the last step. Backward elimination was chosen as the best approach so that insignificant variables were discarded from the model. In backward elimination, all factors are visited in the model, and the least significant is removed. This is repeated until all factors are significant in the resulting model and the bounds of the $95 \%$ confidence interval for the coefficients do not include zero. 
The models correlating the synthetic conditions of $r, \omega$, and [NP] with reverse micelle size for a NP4/NP7 system, as well as for a NP5/NP9 system are presented in Chapter 5. In addition, experiments examining the effects of variables not included in the CCD, i.e., salt concentration, temperature, polar phase, and hydrocarbon phase, on reverse micelle size are also discussed. 


\section{Chapter 5: Results}

\section{Rules of the lab}

1. If an experiment works, something has gone wrong.

2. When you don't know what you're doing, do it neatly.

3. Experiments must be reproducible; they should fail the same way each time.

4. Experience is directly proportional to equipment ruined.

5. Always keep a record of your data. It indicates that you have been working.

6. If you can't get the answer in the usual manner, start at the answer and derive the question.

7. In case of doubt, make it sound convincing.

8. Team work is essential; it allows you to blame someone else.

9. All unmarked beakers contain fast-acting, extremely toxic poisons.

10. Any delicate and expensive piece of glassware will break before any use can be made of it. 


\subsection{Model Results}

Several models were examined as potential predictors of reverse micelle size. Although coefficients and some parameters varied between the models depending on which data were fit, two terms were consistently found in all the models - $\omega$ and $\omega[\mathrm{NP}]$. The best fit model was obtained from the box and star as well as pertinent data from the original star based on statistical analysis, i.e., correlation coefficients, goodness-of-fit tests, and lack-of-fit tests. Table 5.1 provides the data for the final model for the NP4/NP7 system containing three significant variables: the main effect of $\omega$ and the interactive effects of $\mathrm{r} \omega$ and $\omega[\mathrm{NP}]$.

Table 5.1: Model coefficients and results from backward elimination method in SPSS demonstrating pvalues and $95 \%$ confidence intervals.

\begin{tabular}{|c|c|c|c|c|c|}
\hline & \multicolumn{2}{|c|}{ Coefficients } & \multirow{2}{*}{ Significance } & \multicolumn{2}{c|}{$95 \%$ Confidence Interval } \\
\cline { 3 - 6 } & B & Std. Error & & Lower Bound & Upper Bound \\
\hline Constant & 1.75 & 0.93 & 0.040 & 0.15 & 3.6 \\
\hline $\mathrm{r} \omega$ & -3.4 & 1.4 & 0.000 & -6.2 & -4.7 \\
\hline$\omega$ & 3.64 & 0.51 & 0.021 & 2.6 & 0.55 \\
\hline$\left[\mathrm{H}_{2} \mathrm{O}\right]$ & -4.64 & 0.80 & 0.000 & -6.3 & -3.0 \\
\hline
\end{tabular}

From this point on the term $\omega[\mathrm{NP}]$ will be rewritten as $\left[\mathrm{H}_{2} \mathrm{O}\right]$ for simplicity. The following equation demonstrates this equivalence.

$$
\omega[N P]=\left(\frac{\text { molH }_{2} \mathrm{O}}{\text { molNP }}\right)\left(\frac{\text { molNP }}{\text { totalvolume }}\right)=\frac{\text { mol }_{2} \mathrm{O}}{\text { totalvolume }}=\left[\mathrm{H}_{2} \mathrm{O}\right]
$$

The overall empirical formula for the model of NP4/NP7 micelle size prediction is shown in the following equation where $\mathrm{N}=33, \mathrm{R}^{2}=0.84$, and $\mathrm{s}_{\mathrm{R}}=1.50 \mathrm{~nm}$.

$$
\mathrm{Size}=1.75( \pm 0.93)-3.4( \pm 1.4) \mathrm{r} \omega+3.64( \pm 0.51) \omega-4.64( \pm 0.80)\left[\mathrm{H}_{2} \mathrm{O}\right]
$$

The coefficients in Table 5.1 are the coefficients of the specified variables in the model and are shown with their respective standard errors. As discussed earlier, the $95 \%$ 
confidence interval bounds do not include zero for these variables. The p-value is compared to the alpha level (typically 0.05 ) and, if smaller, it can be concluded that the independent variables significantly contributes to the prediction of the dependent variable. All four p-values displayed in Table 5.1 are below the alpha level of 0.05 ; therefore, each variable contributes to explaining some of the experimental variance. The model has been evaluated using the experimental data (Tables 4.1 and 4.2) used to generate the model as well as 10 additional validation points (shown in Table 5.2), and yielded a $\chi^{2}$ goodness-of-fit of 8.70 , which indicates that this is an adequate model at the $99.9 \%$ confidence level. ${ }^{32}$

Table 5.2: Validation experiments to test the model for NP4/NP7 reverse micelle size.

\begin{tabular}{|c|c|c|c|c|c|c|c|}
\hline Solution & $\mathbf{r}$ & $\boldsymbol{\omega}$ & [NP] & Ave Size (nm) & Std Dev & Predicted Size (nm) & \% Error \\
\hline Validation 1 & 0.23 & 6.5 & 0.38 & 7.10 & 0.04 & 8.37 & 17.8 \\
\hline Validation 2 & 0.23 & 6.5 & 0.43 & 7.16 & 0.28 & 7.57 & 5.76 \\
\hline Validation 3 & 0.23 & 5.0 & 0.38 & 6.40 & 0.14 & 6.91 & 7.90 \\
\hline Validation 4 & 0.23 & 5.0 & 0.43 & 6.12 & 0.05 & 6.30 & 2.95 \\
\hline Validation 5 & 0.26 & 6.5 & 0.38 & 10.0 & 0.55 & 8.25 & -17.7 \\
\hline Validation 6 & 0.26 & 6.5 & 0.44 & 6.56 & 0.11 & 7.46 & 13.7 \\
\hline Validation 7 & 0.26 & 5.0 & 0.38 & 6.89 & 0.51 & 6.82 & -0.90 \\
\hline Validation 8 & 0.26 & 5.0 & 0.44 & 6.27 & 0.53 & 6.21 & 0.96 \\
\hline Validation 9 & 0.25 & 6.0 & 0.40 & 6.90 & 0.18 & 7.50 & 8.67 \\
\hline Validation 10 & 0.25 & 6.0 & 0.40 & 7.49 & 0.55 & 7.51 & 0.33 \\
\hline
\end{tabular}

The experimental measurements of reverse micelle size were compared to the modelpredicted values and yielded an average accuracy of $12 \%$. As mentioned earlier, the DLS method has a $10 \%$ error associated with it and the reverse micelle technique, although providing a narrow size distribution, has approximately an $8 \%$ standard deviation ${ }^{28}$, for an overall error of the methodology of $\pm 13 \%$. 
As previously mentioned, NP5/NP9 reverse micelles were also studied in order to evaluate the effects of surfactant chain length on reverse micelle size. Analogous to the NP4/NP7 system, microemulsion solubilization experiments previously discussed were conducted to determine the location of the micellar region in the phase space. This was followed by a similar CCD to determine a model that predicts reverse micelle size from the same parameters. The overall empirical formula for the model of NP5/9 micelle size prediction is shown in the following equation where $\mathrm{N}=23, \mathrm{R}^{2}=0.70$ and $\mathrm{s}_{\mathrm{R}}=7.91$.

$$
\text { Size }=6.9( \pm 4.2)+9.0( \pm 1.7) \omega-15.3( \pm 3.5)\left[\mathrm{H}_{2} \mathrm{O}\right]
$$

\subsection{Effects of Chain Length}

NP5/9 reverse micelles are affected by the same parameters as the NP4/7 but produce larger sized micelles. This trend can be explained by the fact that NP5/9 chains have more oxyethylene groups than NP4/7 chains. As discussed in Chapter 2, hydrogen bonding between the surfactant head groups with themselves or with the water pool controls the stability of the micelle; therefore, the NP5/9 micelles, having more oxyethylene groups, are more stable, allowing them to grow larger. Although, a NP7/9 system would have even more oxyethylene groups, homogeneous micelles did not form with this system. This could be because the NP7 molecules are too large to fill in the gaps between NP9 molecules. Because of this, there is less hydrogen bonding between the surfactant head groups, adding to the instability of this microemulsion.

The different solubilities of water in the two systems is due to the spontaneous curvatures (discussed in Chapter 2) associated with distinct types of surfactants. For NP, 
the surfactant layer always bends toward the water pool. ${ }^{84}$ This provides a greater extent of hydration for the surfactant head group and therefore increases the curvature of the NP4-based micelles. With a sharper surface curvature, micelle pools become smaller and therefore accommodate less water.

Note that the model determined for the NP5/NP9 system shows the reverse micelle size has no dependence on $\mathrm{r}$. It has been suggested that since the tail and head groups of NP5 are comparable in size, the average curvature of the surfactant becomes virtually zero. $^{34}$ As a consequence, the NP5 droplets are prone to agglomerate, forming interdroplet open water channels, and ultimately becoming large water pools. Previous research has demonstrated a concurrence of these interdroplet channels and large water pools for various other microemulsions. ${ }^{85,86}$ This in part explains why the NP5/NP9 system has no dependence on the variable $r$.

\subsection{Effects of r $\omega$}

The $\mathrm{r} \omega$ term in the NP4/NP7 model is an interaction term meaning that the effects of $r$ scale with changes in $\omega$. The negative coefficient indicates that as the product $r \omega$ is increased, the size of the micelle decreases. This is seen in Figure 5.1 where micelle size decreases with increasing $r$, while $\omega$ and [NP] are held constant. 


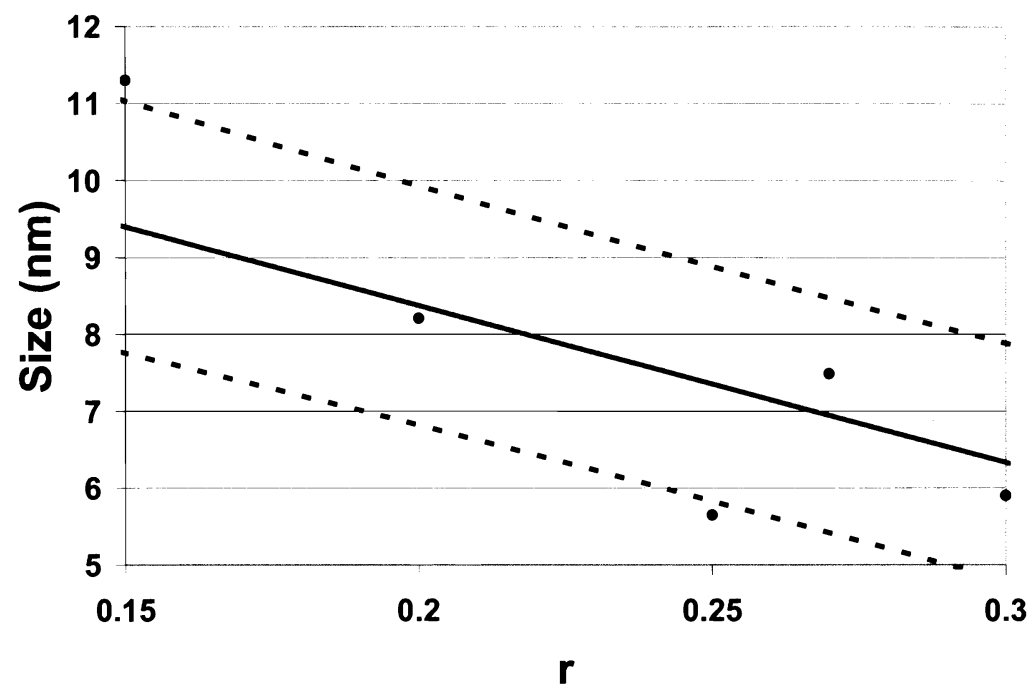

Figure 5.1: Effects of $r$ on NP4/7 reverse micelle size where $\omega=6$ and $[\mathrm{NP}]=0.4$. The dotted lines represent standard error of the model predictions, and the equation for the model is represented by the solid line.

Because stable micelles across a useful range of sizes will not form when using just NP4, one possible explanation of this trend is that the ratio of NP4 to NP7 needed to form micelles is fixed. As $r$ is decreased, the relative concentration of NP7 is increased. In this hypothesis, the equilibrium between free surfactant and micelles would shift, and excess NP4 would be dissolved in the oil phase. However, this would decrease the amount of surfactant used in micelle formation and would result in larger micelles.

A more plausible explanation is based on the curvature of the surfactant layer on the micelles. Recall from Chapter 2 that there are differences in the interaction of each surfactant along the water pool interface. NP7 forms a narrower cone angle from the surface than the corresponding NP4 because the longer length of NP7 molecules allows them to pack closer together. As previously mentioned, NP7 has more oxyethylene groups than NP4, therefore requiring more waters of hydration. This also explains the 
interaction with $\omega$ since the stability of droplets is primarily controlled by the hydrogenbonding association of the surfactant's head groups among themselves and/or with the aqueous cores of droplets.

A three-dimensional cross section of the model for reverse micelle size as a function of $r$ and $\omega$ is displayed in Figure 5.2 with [NP] as a constant at $0.4 \mathrm{M}$. The angled response implies an interaction effect due to $r$ and $\omega$, as discussed above. The negative correlation on micelle size with the interactions of $\omega$ with $r$ is evident. Figure 5.2 indicates that the calculated reverse micelle sizes are in the range of 4 to $13 \mathrm{~nm}$.

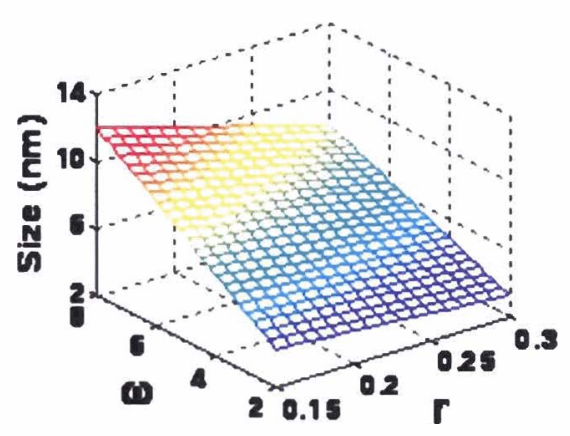

Figure 5.2: Three-dimensional cross section of the model for predicting reverse micelle size from the experimental conditions. $r$ and $\omega$ axes are surfactant to total surfactant ratio and water to surfactant molar ratio, respectively. This cross section is for a constant [NP] of $0.4 \mathrm{M}$.

In addition to hydrogen bonding, since NP7 molecules are able to pack closer together than NP4 molecules, there is less oil penetration between molecules. ${ }^{63-65}$ Therefore, if there are more NP4 molecules, i.e., a larger $r$ value, there is more oil penetration resulting in a smaller sized reverse micelle. Fogler, et al, examined the effects of oxyethylene length on the hydrodynamic diameter of NP reverse micelles in heptane to synthesize silica nanoparticles. ${ }^{34}$ Based on electrical conductivity results, they 
found that the strength of the association of surfactants with droplets increases with the number of oxyethylene groups. Therefore, NP7 molecules adsorb more strongly to water droplets than NP4, forming larger micelles with a smaller $r$. Another possible theory is based on the entire hydrophilic portion sticking into the water pool. Zhong, et al. found that there is one water molecule bound to each OE group. ${ }^{33}$ Since NP7 has more OE groups, i.e., a larger head, it takes up more space for the same number of water molecules.

\subsection{Effects of $\omega$}

Figure 5.3 shows the strong effect of $\omega$ on NP4/NP7 reverse micelle sizeincreasing $\omega$ with other factors kept constant increases micelle size. When water is added to empty reverse micelles, a portion of the water goes to the interface and hydrates the head groups until they reach a maximum becoming fully hydrated at a certain $\omega$. Additional water then goes primarily to the inner core, leading to a continuous increase in the volume of core water with a further increase in $\omega$. This requires a shift in the distribution of surfactant molecules to cover the increased volume of water. As a result, smaller micelles are combined into larger ones. In addition, the effect of $\omega$ on the size of reverse micelles can be explained in terms of the surface area to volume ratio. The surface area of the surfactant shell (SA) can be defined as the product of the number of surfactant molecules and the area of a surfactant head group. Likewise, the volume of the water pool can be defined as the product of the number of water molecules and the volume of a single water molecule. As shown below, the ratio of the two can be reduced 
to the water to surfactant molar ratio. This shows the dependence of reverse micelle size on this ratio.

$$
\begin{aligned}
& S A=4 \pi r^{2}=N_{A}\left(m o l_{\text {surfactimi }}\right)\left(A_{\text {aurficelmant head }}\right) \\
& V=\frac{4}{3} \pi r^{3}=N_{A}\left(m o l_{\mathrm{H}_{2} \mathrm{O}}\right)\left(V_{\mathrm{H}_{2} \text { Onolecule }}\right)
\end{aligned}
$$

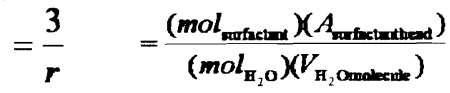

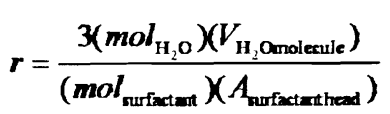

$$
\begin{aligned}
& \omega=K \frac{m o l_{\mathrm{H}_{2} \mathrm{O}}}{\boldsymbol{m o l}_{\text {surfactant }}}
\end{aligned}
$$

These results are consistent with Zhong, et. al.'s evaluation of the effect $\omega$ has on NP reverse micelle size. ${ }^{33}$ Their FT-IR results showed that water droplets in NP reverse micelles become more bulk-like as $\omega$ increases. ${ }^{33}$

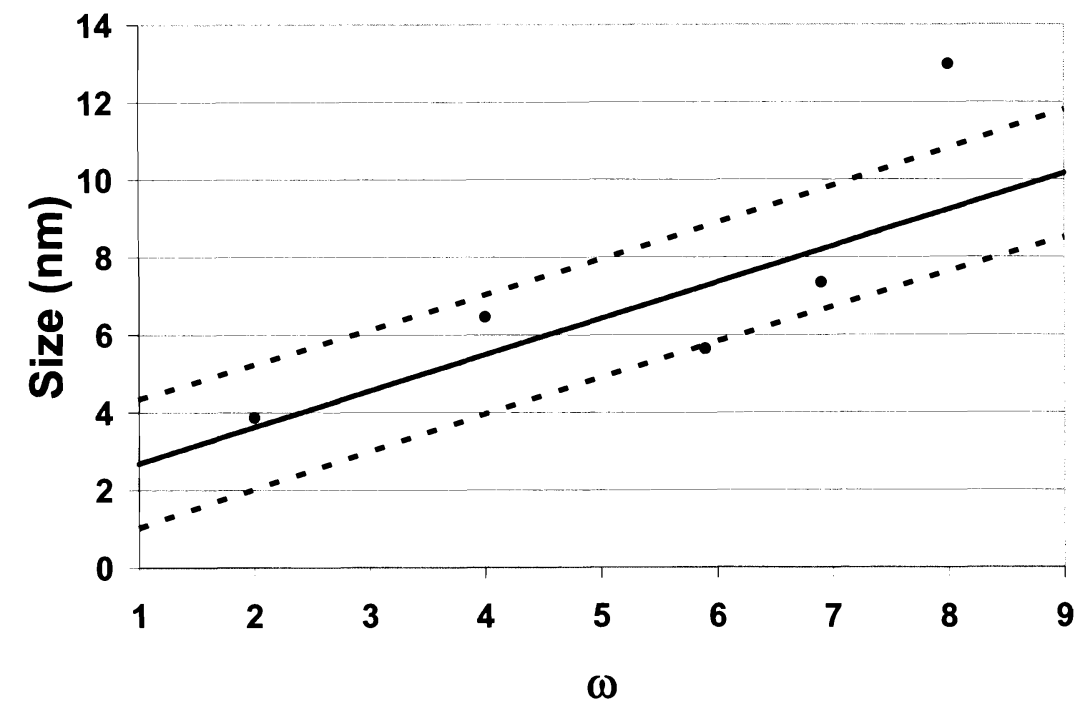

Figure 5.3: Effects of $\omega$ on NP4/7 reverse micelle size where $r=0.25$ and $[\mathrm{NP}]=0.4$. The dotted lines represent standard error of the model predictions, and the equation for the model is represented by the solid line. 


\subsection{Effects of Water Concentration}

Recall from Chapter 4 that water concentration can be derived from surfactant concentration. In order to investigate the main effect of surfactant concentration on reverse micelle size without instigating a change in $\omega$, the amount of cyclohexane was adjusted to alter the surfactant concentration. Micelle size as a function of surfactant concentration is shown in Figure 5.4, illustrating that micelle size increases with a decrease in surfactant concentration when $\omega$ and $\mathrm{r}$ are held constant.

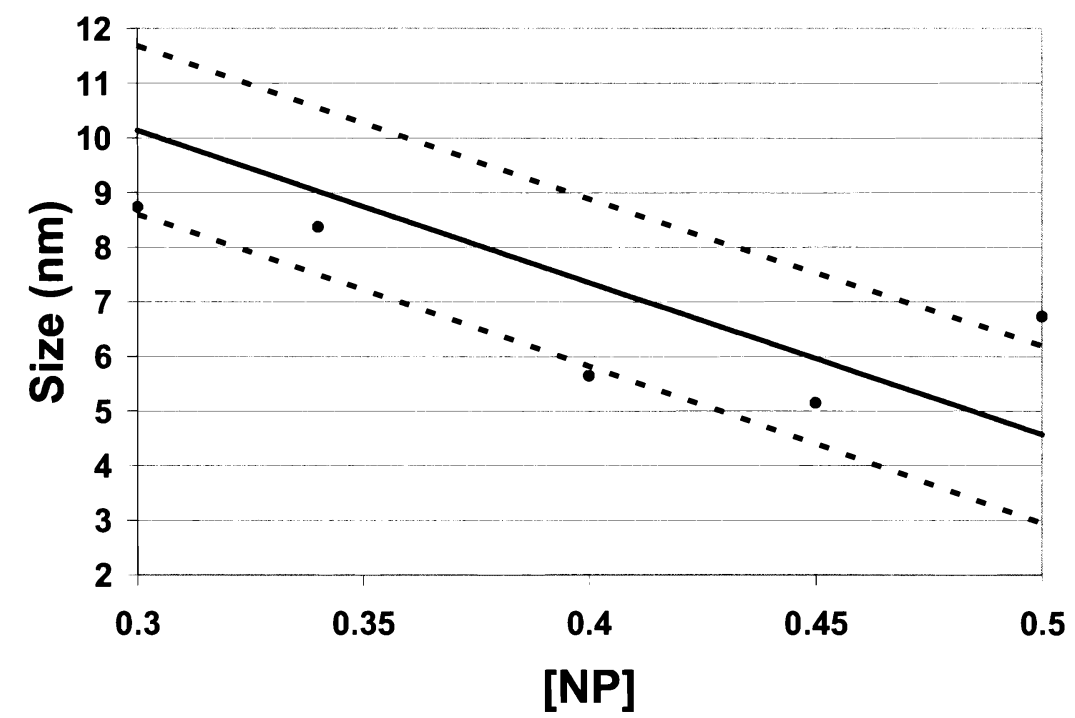

Figure 5.4: Effect of [NP] on NP4/7 reverse micelle size where $r=0.25$ and $\omega=6$. The dotted lines represent standard error of the model prediction, and the equation for the model is represented by the solid line.

As mentioned earlier, the model term containing $\omega$ and $[\mathrm{NP}]$ can be rewritten as $\left[\mathrm{H}_{2} \mathrm{O}\right]$. This is easily understood by altering the concentration of cyclohexane; increasing the oil phase will decrease both $[\mathrm{NP}]$ and $\left[\mathrm{H}_{2} \mathrm{O}\right]$. With the addition of cyclohexane, the surfactant-micelle equilibrium is shifted because more surfactant molecules can be dissolved in the oil phase. This means that the number of free surfactant molecules is 
increased. Consequently the number of surfactant molecules involved in micelle formation (i.e., the aggregation number) is decreased. Micelle size is primarily influenced by the relationship between the volume of water and the surface area of aggregated surfactant. When decreasing the number of aggregated surfactant molecules, the micelles will coalesce to form larger micelles. This is evident from the model given that it predicts that decreasing the $\left[\mathrm{H}_{2} \mathrm{O}\right]$ while holding $\omega$ constant will result in larger micelles.

Figure 5.5 shows strong effects of $[\mathrm{NP}]$ and $\omega$ on reverse micelle size with the $\mathrm{r}$ factor constant at 0.25 . The angled response implies an interaction effect due to [NP] and $\omega$ (in other words, $\left[\mathrm{H}_{2} \mathrm{O}\right]$ ), which is consistent with the model found. This surface shows similar results as shown in Figure 5.2 for $\mathrm{r}$ and $\omega$. The negative correlation on micelle size with the interactions of $\omega$ with both $r$ and $[N P]$ are evident from these figures.

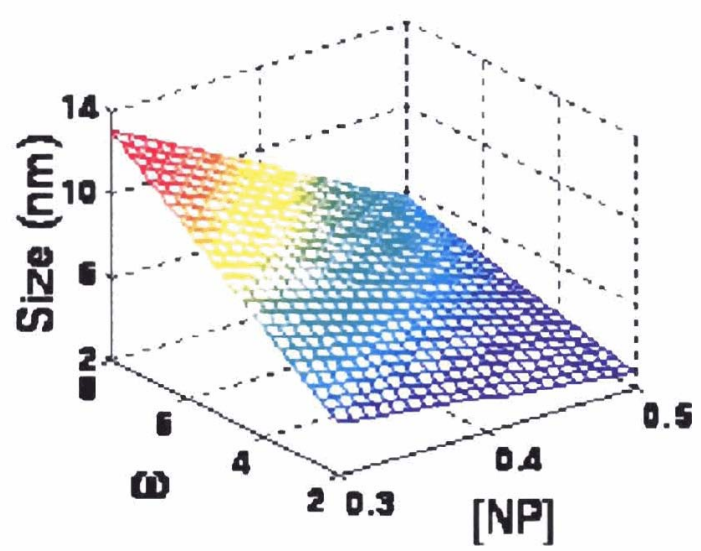

Figure 5.5: Three-dimensional cross section of the model for predicting reverse micelle size from the experimental conditions. [NP] and $\omega$ axes are surfactant concentration and water to surfactant molar ratio, respectively. This cross section is for a constant $r$ of $0.25 \mathrm{M}$. 
Berger et al. used DLS and tensiometry measurements to show that micelle size is sensitive to surfactant concentration. ${ }^{87}$ In addition, Haldar et. al. demonstrated similar results with the single-headed cationic surfactant cetylpyridinium bromide. ${ }^{88}$ They also attribute this decrease in size to an increased aggregation number at higher surfactant concentrations.

\subsection{Salt Concentration}

In order to investigate the influence of salts on NP reverse micelles, microemulsion experiments were performed in the presence of $\mathrm{NaCl}$. The concentration of $\mathrm{NaCl}$ was varied while $\mathrm{r}, \omega$, and NP were held constant. Although some research has suggested salt concentration within reverse micelles might affect the hydrodynamic size, there is no apparent NP micelle size-dependence on $\mathrm{NaCl}$ concentration as seen in Figure 5.6.

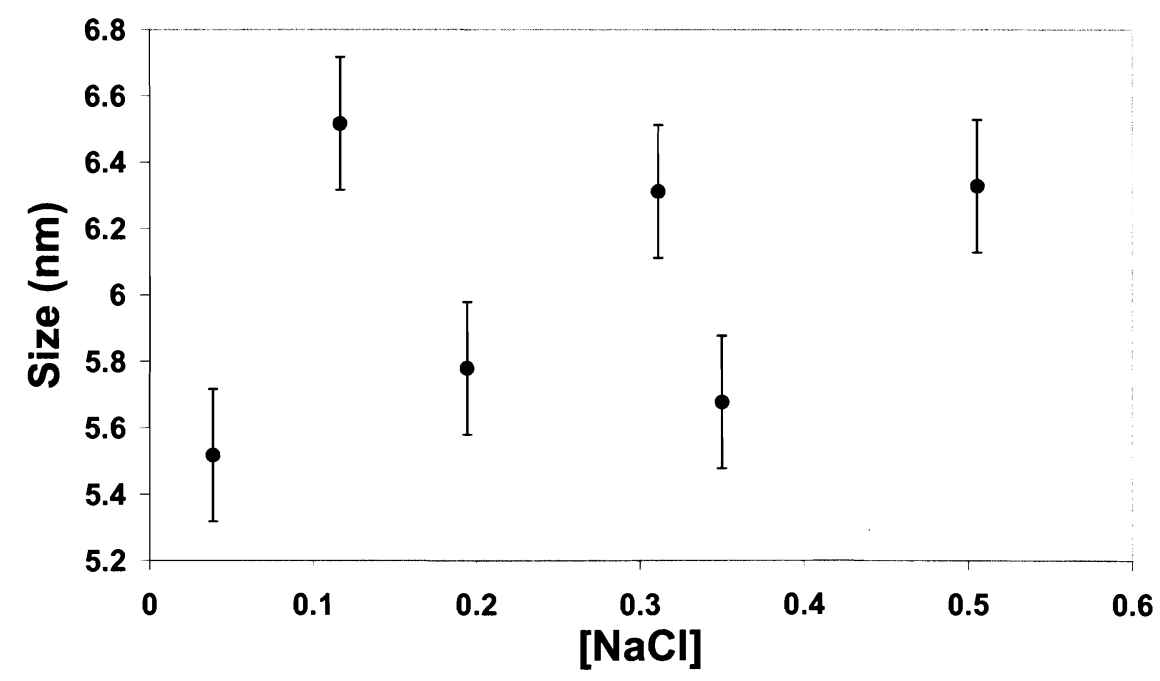

Figure 5.6: Reverse micelle size dependence on $\mathrm{NaCl}$ concentration. All points fall within standard deviations of each other indicating no dependence. 
This is most likely dependent on the type of salt within the water pool, i.e., whether it affects the hydrogen bonding network with the head groups or not. If the salt within the water pool weakens the hydrogen bonding of water to the surfactant's polyoxyethylene head group, the droplets are smaller than with pure water. This effect is not seen with the addition of $\mathrm{Na}$ and $\mathrm{Cl}$ ions to the micelles since there is no weakening of hydrogen bonds. Studies of $\mathrm{AOT}^{89}$ as well as $\mathrm{NP}^{34}$ reverse micelles show that added electrolytes such as ammonia can reduce the $\mathrm{CMC}$ or aggregation number for this reason. This weakening of the hydrogen bonds decreases the hydration of the head groups, which eventually results in the formation of smaller droplets. ${ }^{34}$ In addition, time-resolved fluorescence quenching results reported by Sando, et al. indicate that azide ions dissolved in NP reverse micelles (up to $2.3 \mathrm{M}$ ) have no observable influence on $\mathrm{CMC}$ and their aggregation behavior. ${ }^{90}$

\subsection{Temperature}

Keeping the surfactant concentration, $r$, and $\omega$ fixed at $0.4 \mathrm{M}, 0.25$, and 6 respectively, DLS measurements were performed at different temperatures ranging from 20 to $30^{\circ} \mathrm{C}$. Figure 5.7 shows strong effects of temperature on micelle size with all other factors kept constant. 


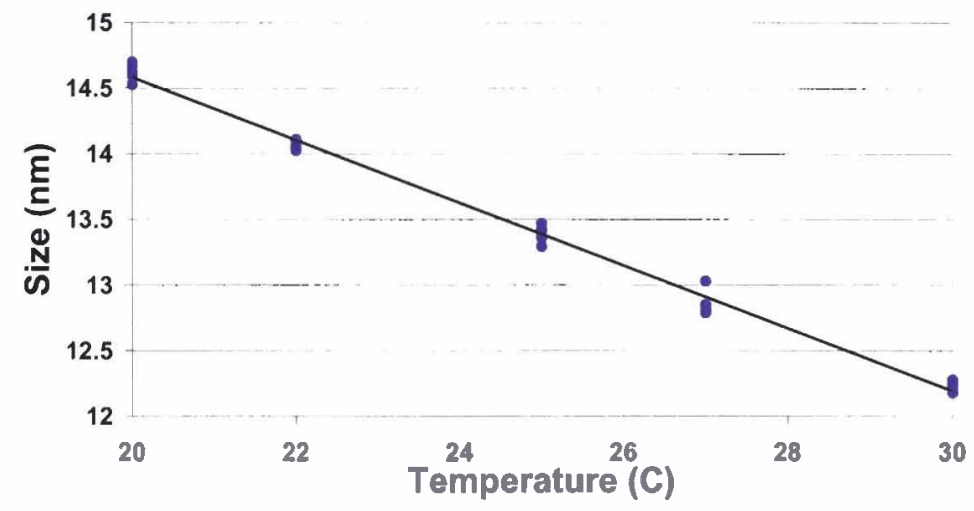

Figure 5.7: Temperature-dependence of NP4/7 reverse micelle size corrected for viscosity where $r=0.25$, $\omega=6$, and $[\mathrm{NP}]=0.4$.

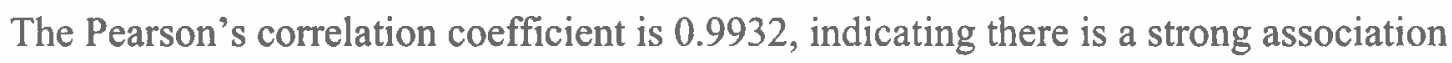
between temperature and reverse micelle size. The negative correlation between the two demonstrates that an increase in temperature results in a decrease in micelle size. This is as expected since polyethoxylates exhibit an inverse temperature-solubility relationship. ${ }^{37}$ That is, as the solution temperature is increased, the NP solubility in water decreases. This phenomenon is attributed to a disruption of specific interactions, i.e., hydrogen bonding, between the water and the polyethoxylate units in the molecule. In addition, an increase in temperature leads to a higher CMC. This results in a decrease in reverse micelle size. Generally for nonionic surfactants, temperature is a critical parameter due to the strong dependence of their solubility (in water or oil) on temperature. For surfactants of the PEO type, as temperature increases water solvation is weakened for the hydrophilic units and penetrates less into the surfactant layer. In addition, on the other side of the interface, oil can penetrate further into the hydrocarbon chains, so that increasing temperature for this type of surfactant causes a strong decrease in $\mathrm{C}_{0}$ as 
discussed in Section 2.8. This phenomenon explains the strong temperature effects on the size of NP reverse micelles.

Though studies on the temperature dependence of NP micelle size have yet to be reported, previous studies on other surfactants such as Triton X-100, Brij-35, and

pyridinium surfactants that are dependent on temperature have been reported. ${ }^{6,31,88,91-93}$ While temperature does in fact affect micelle size, it was not included as a parameter in the determination of the model. This is because micelle size can be easily manipulated with temperature alone and the interaction behavior of temperature with the other independent variables may be highly complex.

\subsection{Polar Phase Effects}

The stability of reverse micelles is dependent on the nature of the polar solvent. Much is known about reverse micelles solubilizing water, however many reactions scaled down to the nanosize scale occur when using nonaqueous polar solvents. For many reactions, water is not the solvent of choice as the medium in which the reaction occurs; therefore it is attractive to isolate solvents other than water inside micelles. These systems have some potential practical use in organic reactions like esterification and polymerization. Furthermore, it is desirable to examine the nature of the micelle without the effects of hydrogen bonding from water; therefore, in addition to water, the use of formamide as the polar solvent was investigated.

Analogous to the data obtained for water, micelle size increased only with increasing $\omega$ and decreasing [NP]. Formamide-containing micelles are in general much 
larger than aqueous micelles with similar $\omega$ and $[\mathrm{NP}]$. For example, NP/water micelles with $\mathrm{r}=0.25, \omega=1.2$, and $[\mathrm{NP}]=0.40$ have sizes of less than $4 \mathrm{~nm}$ whereas $\mathrm{NP} /$ formamide micelles with $\mathrm{r}=0.25, \omega=1.9$, and $[\mathrm{NP}]=0.40$ have sizes over $16 \mathrm{~nm}$ (see Table 5.3). This is due to the more pronounced aggregation tendency, i.e., a larger aggregation number, of the surfactants with more apolar solvents. This follows the trend of increased reverse micelle size with less hydrogen bonding with the head groups.

Table 5.3: Effects on NP reverse micelle size by changing the polar phase to formamide

\begin{tabular}{|c|c|c|c|c|c|}
\hline Solution & $\mathbf{r}$ & $\boldsymbol{\omega}$ & [NP] & Ave Size (nm) & Std Dev \\
\hline 1 & & & & & \\
\hline 2 & $\mathbf{0 . 1 5}$ & 0.6 & 0.40 & 15.11 & 0.31 \\
\hline 3 & $\mathbf{0 . 2 5}$ & 0.6 & 0.40 & 10.24 & 0.25 \\
\hline 4 & $\mathbf{0 . 3 1}$ & 0.6 & 0.41 & 9.59 & 0.13 \\
\hline 5 & $\mathbf{0 . 3 5}$ & 0.6 & 0.41 & 8.60 & 0.48 \\
\hline 6 & $\mathbf{0 . 4 0}$ & 0.6 & 0.41 & 7.78 & 0.21 \\
\hline 7 & 0.25 & 0.6 & $\mathbf{0 . 2 1}$ & 11.37 & 0.22 \\
\hline 8 & 0.25 & 0.6 & $\mathbf{0 . 3 0}$ & 10.95 & 0.22 \\
\hline 9 & 0.25 & 0.6 & $\mathbf{0 . 4 0}$ & 10.49 & 0.41 \\
\hline 10 & 0.25 & 0.6 & $\mathbf{0 . 5 0}$ & 10.01 & 0.09 \\
\hline 11 & 0.25 & 0.6 & $\mathbf{0 . 6 0}$ & 9.70 & 0.19 \\
\hline 12 & 0.25 & $\mathbf{1 . 2}$ & 0.40 & 16.27 & 0.55 \\
\hline 13 & 0.25 & $\mathbf{1 . 0}$ & 0.40 & 14.76 & 0.18 \\
\hline 14 & 0.25 & $\mathbf{0 . 8}$ & 0.40 & 12.97 & 0.22 \\
\hline 15 & 0.25 & $\mathbf{0 . 6}$ & 0.40 & 11.91 & 0.15 \\
\hline & 0.25 & $\mathbf{0 . 4}$ & 0.40 & 10.71 & 0.72 \\
\hline
\end{tabular}

These results agree with the work of Levinger and Molina-Bolivar, who also found small values for $\omega$ (below 2) and showed it is possible to create microenvironments with nonaqueous polar solvents. ${ }^{94,95}$ Other research has shown that poor solvation of AOT polar heads by formamide is responsible for the novel features exhibited by these microemulsions, i.e., large variations of AOT micelle size with both temperature and $\omega$. Nonionic surfactants encapsulating formamide, however, show a phase behavior identical 
to that displayed by water in-oil systems, although the amount of polar solvent that it is possible to encapsulate in the latter is smaller and percolation happens at lower temperatures. ${ }^{96}$

\subsection{Hydrocarbon Phase Effects}

The type of solvent used for reverse micelles can influence the transfer of surfactant molecules from the aqueous phase to the organic phase. The solvents that can be used in reverse micelle systems must be immiscible with water. ${ }^{70,97}$ Hexane, benzene, and isooctane were examined as various solvents in addition to cyclohexane. Oil molecules with smaller sizes or higher polarities are able to penetrate into surfactant films around water/oil micelle pools causing size reduction and morphological change. This explains why hexane and isooctane do not form stable spherical reverse micelles with water since they have small, but significant dipole moments and are slightly miscible with water relative to cyclohexane. Using benzene as the oil phase did not result in the formation of reverse micelles either, although this solvent is nonpolar and should remain separate from the water. We attribute this to aromatic stacking between the oil and surfactant. NP molecules contain substituted benzene rings in the center of their chain; therefore, in binding to the solvent, benzene would disrupt the lamellar layering of surfactant chains necessary to form micelles. Chang and Chen reported the influence of several solvents on trypsin extraction into aqueous reverse micelles. Using isooctane, octane, heptane, hexane, cyclohexane and kerosene as solvents, they observed that a higher percentage of transfer (about 70\%) occurred with kerosene, whereas with cyclohexane this value decreased to $35 \% .{ }^{45,97}$ Kadam stated that this effect occurs 
because all these solvents are capable of denaturing the reversed micelle structure. ${ }^{98}$ Fogler also found similar results investigating the effects of the type of oil (heptane and cyclohexane) on some of the same nonionic surfactants used here. ${ }^{34}$ 


\section{Conclusions}

A group of organic molecules were having a party, when a group of robbers broke into the room and stole all of the guest's joules. A tall, strong man, armed with a machine gun came into the room and killed the robbers one by one. The guests were very grateful

to this man, and they wanted to know who he was. He replied: "My name is BOND,

\section{Covalent Bond."}


Analysis of the effects from possible experimental variables indicates that the best approach for size control is varying temperature, water concentration, and the water to surfactant molar ratio, as well as the proportion of short and long chain surfactants. The resulting change in micelle size due to changes in $r, \omega$, and $[\mathrm{NP}]$ are different. This allows the system to be highly tunable. Adjusting $\omega$ will yield large changes of predictable micelle size while adjusting the water concentration and $\mathrm{r}$ will give smaller, predictable changes in micelle size. In addition, reverse micelle size can be fine tuned via temperature.

Clearly, the multivariate model developed in this work provides an excellent means to predict reverse micelle size from 4 to $13 \mathrm{~nm}$ with a precision of $\pm 2 \mathrm{~nm}$, with the greater error present at the lower sizes due to the complex equilibrium between free surfactant molecules and micellar structures. This model shows that the prediction of micelle size is achievable by using the statistical method of factorial design to quantitatively correlate the size with the synthetic conditions. Such a model is essential for producing nanoparticles with a targeted size on industrial scales that have particular desired properties as well as offering the ability to optimize and balance conflicting processing constraints in order to achieve a particular size. These results suggest that a combination of NP based surfactants can be successfully engineered to control the extent of formation and the structure of reverse micelles. This is especially useful for producing materials on the nanoscale where there exists the possibility that surface based properties, magnetism, and electronic properties may be enhanced by controlling the particle size. 


\section{Appendix}

\section{You Might Be a Chemist if...}

you carry your lab safety goggles around with you at all times, just in case...

* you don't drink water, you drink $\mathrm{H}_{2} \mathrm{O}$.

* you start disagreeing with movies and TV shows on scientific aspects.

* you carry a base solution around with you at all times, just in case one of those freak hydrochloric acid spills happen.

* you become very agitated when people refer to air as oxygen, and proceed to list all of the components of air.

* instead of writing ozone you write $\mathrm{O}_{3}$.

* you start referring to the smell of nail polish remover as an acetone smell.

* you actually enjoy going to Chemistry class.

* you think a mole is a unit of amount, rather than a small furry animal in your lawn.

* you pronounce unionized as "un-ion-ized", instead of "union-ized".

* you wash your hands before you go to the bathroom. 
In order to further explore the relationship between synthetic conditions and nanoparticle size, gold nanoparticles were investigated. The synthesis of gold nanoparticles has been studied intensively for their potential use in a diverse range of applications from sensors for heavy metal ions ${ }^{99,} 100$ and electronic nanodevices ${ }^{101}$ to biological tagging. ${ }^{102,103}$ Since reactions such as reduction for nanoparticle synthesis can be done within a micelle system and reduction of gold is well documented, the system seemed well suited for this investigation.

Gold colloids were synthesized via borohydride reduction of gold chloride by both aqueous phase exchange and coalescence. Nanoparticles larger than $5 \mathrm{~nm}$ tend to aggregate due to van der Waals attractions. Therefore, stabilization by a thiol was attempted to prevent agglomeration and produce dispersed particles. ${ }^{104}$ Although many attempts were made with various reductants, concentrations, and at different $\mathrm{pH}$ 's, correlating the size of gold nanoparticles with the models for reverse micelle size generated here was unsuccessful due to premature reduction of gold by NP. 


\section{References}

1. Suib, S. L.; Zhang, Z., Journal of the American Chemical Society 1988, 110, (16), 5569-5571.

2. Sun, S.; Zeng, H., Journal of the American Chemical Society 2002, 124, 82048205 .

3. Yonezawa, T.; Yasui, K.; Kimizuka, N., Langmuir 2001, 17, 271-273.

4. Wright, D. W.; Knecht, M. R., Chem. Mater. 2004, 16, 4890-4895.

5. Wang, H.; Tashiro, A.; Nakamura, H.; Uehara, M.; Miyazaki, M.; Watari, T.; Maeda, H., Journal of Materials Research 2004, 19, (11), 3157-3161.

6. Kim, K. D.; Lee, T. J.; Kim, H. T., Colloids and Surfaces A: Physicochem. Eng. Aspects. 2003, 224, 1.

7. Rondinone, A. J.; Samia, A. C. S.; Zhang, J. Z., J. Phys. Chem. B 2000, 104, 7919.

8. Willard, M. A.; Kurihara, L. K.; Carpenter, E. E.; Calvin, S.; Harris, V. G., International Materials Reviews 2004, 49, (3-4), 125-170.

9. Hochepied, J. F.; Pileni, M. P., Journal of Applied Physics 2000, 87, (5), 24722478.

10. Feldmann, C.; Jungk, H. O., Angewandte Chemie-International Edition in English 2001, 40, (2), 359-362.

11. Massart, R., IEEE Transactions on Magnetics 1981, 17, (2), 1247-1248.

12. Huang, C. L.; Matijevic, E., Solid State Ionics 1996, 84, 249-258.

13. Abu Mukh-Qasem, R.; Gedanken, A., Journal of Colloid and Interface Science 2005, 284, (2), 489-494.

14. Sun, S. H.; Zeng, H.; Robinson, D. B.; Raoux, S.; Rice, P. M.; Wang, S. X.; Li, G. X., Journal of the American Chemical Society 2004, 126, (1), 273-279.

15. Jonsson, B. J.; Turkki, T.; Strom, V.; El-Shall, M. S.; Rao, K. V., Journal of Applied Physics 1996, 79, (8), 5063-5065. 
16. Nawathey-Dikshit, R.; Shinde, S. R.; Ogale, S. B.; Kulkarni, S. D.; Sainkar; R., S.; Date, S. K., Applied Physics Letters 1996, 68, (24), 3491-3493.

17. Jiang, J. S.; Gao, L.; Yang, X. L.; Guo, J. K.; Shen, H. L., Journal of Materials Science Letters 1999, 18, 1781-1783.

18. Jiang, J. S.; Yang, X. L.; Gao, L.; Guo, J. K.; Jiang, J. Z., Nanostructure Materials 1999, 12, (143-146).

19. Cushing, B. L.; Kolesnichenko, V. L.; O'Connor, C. J., Chemical Review 2004, 104, (9), 3893-3946.

20. Jeyadevan, B.; Tohji, K.; Nakatsuka, K.; Narayanasamy, A., Journal of Magnetism and Magnetic Materials 2000, 217, (1-3), 99-105.

21. Auzans, E.; Zins, D.; Blums, E.; Massart, R., Journal of Materials Science 1999, 34, 1253-1260.

22. Glavee, G. N.; Klabunde, K. J.; Sorensen, C. M.; Hadjipanayis, G. C., Langmuir 1994, 10, (12), 4726-4730.

23. Glavee, G. N.; Klabunde, K. J.; Sorensen, C. M.; Hadjipanayis, G. C., Inorganic Chemistry 1995, 34, (1), 28-35.

24. Lee, H. S.; Lee, W. C.; Furubayashi, T., Journal of Applied Physics 1999, 85, (8), 5231-5233.

25. Shirtcliffe, N.; Nickel, U.; Schneider, S., Journal of Colloid and Interface Science 1999, 211, 122-129.

26. Moumen, N.; Pileni, M. P., Chem. Mater. 1996, 8, 1128-1134.

27. Willard, M. A.; Kurihara, L. K.; Carpenter, E. E.; Calvin, S.; Harris, V. G., International Materials Reviews 2004, 49, (3-4), 125-170.

28. Pileni, M. P.; Hammouda, A.; Moumen, N.; Lisiecki, I., Control of the size and shape of nanoparticles. In Fine Particle Science and Technology, Pelizzetti, E., Ed. Kluwer Academic Publishers: Netherlands, 1996; pp 413-429.

29. Burda, C.; Chen, X.; Narayanan, R.; El-Sayed, M. E., Chem. Rev. 2005, 105, (4), 1025-1102.

30. Douglas, E. P.; Jia, W., Journal of Materials Chemistry 2004, 14, 744-751. 
31. Krei, G.; Meyer, U.; Börner, B.; Hustedt, H., Bioseparation 1995, 5, (175-183).

32. Otto, M., Chemometrics: Statistics and Computer Application in Analytical Chemistry. Wiley-VCH: Weinheim, 1999.

33. Zhong, Q.; Owrutsky, J. C.; Steinhurst, D. A.; Carpenter, E. E., Langmuir 2002, $18,7401-7408$.

34. Fogler, H. S.; Chang, C.-L., Langmuir 1997, 13, 3295-3307.

35. Aniansson, E. A. G.; Wall, S. N., J. Phys. Chem. 1974, 78, 1024.

36. Morrison, S. A.; Carpenter, E. E.; Harris, V. G.; Cahill, C. A., Journal of Nanoscience and Nanotechnology 2005, 5, (9), 1323-1344.

37. Meyers, D., Surfactant Science and Technology. 2nd ed.; VCH Publishers: New York, 1992.

38. Luisi, P. L.; Magid, L. J., Crit. Rev. Biochem. 1986, 20, 409-474.

39. Eastoe, J.; Robinson, B. H.; Steytler, D. C.; Thronleeson, D., Advances in Colloid and Interface Science 1991, 36, 1-31.

40. Jain, T. K.; Varshney, M.; Maitra, A., Journal of Physical Chemistry 1989, 93, 7409-7416.

41. Petit, C.; Lixon, P.; Pileni, M. P., Langmuir 1991, 7, 2620-2625.

42. Pileni, M.-P.; Zemb, T.; Petit, C., Chemical Physics Letters 1985, 118, (4), 414420.

43. Yener, D. O.; Giesche, H., J. Am. Ceram. Soc. 2001, 84, (9), 1987-1995.

44. Zulauf, M.; Eicke, H.-F., Journal of Physical Chemistry 1979, 83, (4), 480-486.

45. Chen, Y.-P.; Cheng, J.-S., Fluid Phase Equilibria 2005, 232, 37-43.

46. Flores, M. V.; Voutsas, E. C.; Spiliotis, N.; Eccleston, G. M.; Bell, G.; Tassios, D. P.; Halling, P. J., Journal of Colloid and Interface Science 2001, 240, 277-283.

47. Hsiao, L.; Dunning, H. N.; Lorenz, P. B., J. Phys. Chem. 1956, 60, 657.

48. Wennerström, H.; Soderman, O.; Olsson, U.; Lindman, B., Colloids and Surfaces A: Physicochemical and Engineering Aspects 1997, 123-124, 13-26. 
49. Micelles, Microemulsions, and Monolayers. Marcel Dekker, Inc.: New York, 1998.

50. Nusselder, J. J. H.; B., E. J., J. Colloid Interface Sci. 1992, 148, 353.

51. Paredes, S.; Tribout, M.; Ferreira, J.; Leonis, J., J. Colloid Poym. Sci. 1976, 254, 637.

52. Evans, D. F.; Ninham, B. W., J. Phys. Chem. 1986, 90, 226.

53. Paula, S.; Willy, S.; Tuchtenhagen, J.; Blume, A., J. Phys. Chem. 1995, 99, $11742-11751$.

54. McBain, J. W.; Salmon, C. S., Journal of the American Chemical Society 1920, $43,426$.

55. Evans, D. F.; Wennerstrom, H., The Colloidal Domain. 2nd ed.; Wiley-VCH: New York, 1999.

56. Griffin, W. C., J. Soc. Cosmet. Chem. 1949, 1, 311.

57. Davies, J. T.; Rideal, E. K., Interfacial Phenomena. 2nd ed.; Academic Press: London, 1963.

58. Shinoda, K.; Saito, H., J. Colloid Interface Sci. 1969, 30, 258.

59. Becher, P., Emulsions Theory and Practice. 2nd ed.; Reinhold Publishing Corp.: New York, 1965.

60. Mitchell, D. J.; Ninham, B. W., J. Chem. Soc. Faraday Trans. 2 1981, 77, 601.

61. Israelachvili, J. N., Intermolecular and Surface Forces. Academic Press: London, 1985.

62. Ghosh, S. K.; Khatua, P. K.; Bhattacharya, S. C., Int. J. Mol. Sci. 2003, 4, 562571.

63. Eastoe, J.; Dong, J.; Hetherington, K. J.; Steytler, D. C.; Heenan, R. K., J. Chem. Soc. Faraday Trans. 1996, 92, 65.

64. Eastoe, J.; Hetherington, K. J.; Sharpe, D.; Dong, J.; Heenan, R. K., Langmuir 1996, 12, 3876. 
65. Eastoe, J.; Hetherington, K. J.; Sharpe, D.; Steytler, D. C.; Egelhaaf, S.; Heenan, R. K., Langmuir 1997, 13, 2490.

66. Gan, L. M.; Liu, B.; Chew, C. H.; Xu, S. J.; Chua, S. J.; Loy, G. L.; Xu, G. Q., Langmuir 1997, 13, 6427-6431.

67. Lim, G. K.; Qang, J.; Ng, S. C.; Gan, L. M., Langmuir 1999, 15, 7472-7477.

68. Lim, G. K.; Wang, J.; Ng, S. C.; Chew, C. H.; Gan, L. M., Biomaterials 1997, 18, 1433-1439.

69. Van Hamme, J. D.; Ward, O. P., Can. J. Microbiol. 1999, 45, 130-137.

70. Luisi, P. L.; Giomini, M.; Pileni, M. P.; Robinson, B. H., Biochim. Biophys. Acta. 1988, 47, 209-246.

71. Rosen, M. J.; Zhou, Q., Langmuir 2001, 17, 3532-3537.

72. Schmidt, P. W., Modern Aspects of Small-Angle Scattering. 1995.

73. Zetasizer Nanoseries User Manual; Malvern Instruments Ltd.: Worcestershire, 2003; pp 1-256.

74. Atkins, P.; de Paula, J., Physical Chemistry. 7th ed.; W. H. Freeman and Company: New York, 2002; p 1139.

75. Skoog, D. A.; Holler, F. J.; Neiman, T. A., Principles of Instrumental Analysis. 5th ed.; Brooks/Cole Thomas Learning: 1998.

76. Ishizu, K.; Mori, A., Polymer International 2001, 51, (1), 50-54.

77. Liu, Y.-L.; Lee, H.-T.; Chang, C.-C.; Kan, L.-S., Biochemical and Biophysical Research Communications 2003, 306, 59-63.

78. Thurmond II, K. B.; Remsen, E. E.; Kowalewski, T.; Wooley, K. L., Nucleic Acids Research 1999, 27, (14), 2966-2971.

79. CRC Handbook of Chemistry and Physics. 55 ed.; CRC Press: Cleveland, 1974.

80. Herrera, A. P.; Resto, O.; Briano, J. G.; Rinaldi, C., Nanotechnology 2005, 16, S618-S625.

81. Massart, D. L.; Vandeginste, B. G. M.; Deming, S. N.; Michotte, Y.; Kaufman, L., Chemometrics: A Textbook. Elsevier Science: Amsterdam, 1988; Vol. 2. 
82. Beebe, K. R.; Pell, R. J.; Seasholtz, M. B., Chemometrics: A Practical Guide. Wiley Interscience: New York, 1998.

83. Deming, S. N.; Morgan, S. L., Experimental Desiogn: A Chemometric Approach. Elsevier Science: Amsterdam, 1987; Vol. 3.

84. Caldararu, H.; Caragheorgheopol, A.; Vasilescu, M.; Dragutan, I.; Lemmetyinen, H. J., J. Phys. Chem. 1994, 98, 5320.

85. Jada, A.; Lang, J.; Zana, R., J. Phys. Chem. 1989, 93, 10.

86. Jada, A.; Lang, J.; Zana, R., J. Phys. Chem. 1990, 94, 387.

87. Berger, B. W.; Gendron, C. M.; Robinson, C. R.; Kaler, E. W.; Lenhoff, A. M., Acta. Cryst. 2005, D61, 724-730.

88. Haldar, J.; Aswal, V. K.; Goyal, P. S.; Bhattacharya, S., J. Phys. Chem. B 2004, $108,11406-11411$.

89. Paul, B. K.; Mitra, R. K., Journal of Colliod and Interface Science 2005, 288, 261-279.

90. Sando, G. M.; Dahl, K.; Zhong, Q.; Owrutsky, J. C., Vibrational Relaxation of Azide in Formamide Reverse Micelles. Journal of Physical Chemistry A 2005, $109,5788-5792$.

91. Callaghan, A.; Doyle, R.; Alexander, E.; Palepu, R., Langmuir 1993, 9, (34223426).

92. Kumbhakar, M.; Mukherjee, T.; Pal, H., Photochemistry and Photobiology 2005, $81,(3), 588-594$.

93. Turro, N. J.; Kuo, P.-L., J. Phys. Chem. 1986, 90, (17), 4205-4210.

94. Levinger, N. E.; Riter, R. E.; Kimmel, J. R.; Undiks, E. P., J. Phys. Chem. B 1997, 101, 8292-8297.

95. Molina-Bolivar, J. A.; Aguiar, J.; Peula-Garcia, J. M.; Ruiz, C. C., Molecular Physics 2002, 100, (2), 3259-3269.

96. Laia, C. A. T.; Lo'pez-Cornejo, P.; Costa, S. M. B.; d'Oliveira, J.; Martinho, J. M. G., Langmuir 1998, 14, 3531-3537. 
97. Chang, Q. L.; Chen, J. Y., Biotechnol. Bioeng. 1995, 46, 172-174.

98. Kadam, K. I., Enzyme Microb. Technol. 1986, 8, 266-273.

99. Kim, Y.; Johnson, R. C.; Hupp, J. T., Nano. Lett. 2001, 1, 165.

100. Liu, J.; Lu, Y., J. Am. Chem. Soc. 2003, 125, 6642.

101. Shipway, A. N.; Katz, E.; Willner, I., Chem. Phys. Chem. 2000, 1, 18.

102. Connolly, S.; Cobbe, S.; Fitzmaurice, D., J. Phys. Chem. B 2001, 105, 2222.

103. Khlebtsov, N. G.; Bogatyrev, V. A.; Dykman, L. A.; Melnikov, G. A., J. Colloid Interface Sci. 1996, 180, 436.

104. Balasubramanian, R.; Kim, B.; Tripp, S. L.; Wang, X.; Lieberman, M.; Wei, A., Langmuir 2002, 18, 3676. 


\section{VITA}

Melissa Ann Michaels was born on January 3, 1982 in Princeton, New Jersey. She received her Bachelor of Arts degree in Chemistry from Randolph-Macon Woman's College in Lynchburg, Virginia in May 2003. During her graduate career at Virginia Commonwealth University, she taught as an adjunct professor at Virginia State University. 